\title{
Emerging ultrasound technologies for early markers of disease
}

\author{
Ernest J. Feleppa ${ }^{\mathrm{a}, *}$, S. Kaisar Alam ${ }^{\mathrm{a}}$ and Cheri X. Deng ${ }^{\mathrm{b}}$ \\ ${ }^{a}$ Riverside Research Institute, Biomedical Engineering Laboratories, 156 William Street, New York, NY \\ 10038-2609, USA \\ ${ }^{\mathrm{b}}$ Case Western Reserve University, Department of Biomedical Engineering, 10900 Euclid Avenue, Cleveland, Ohio \\ 44106-7220, USA
}

\begin{abstract}
Ultrasound has been a popular clinical imaging modality for decades. It is well established as a means of displaying the macroscopic anatomy of soft-tissue structures. While conventional ultrasound methodologies (i.e., B-mode and Doppler methods) are well proven and continue to advance technically on a daily basis, e.g. by extending into higher frequencies and taking advantage of harmonic phenomena in tissues, fundamentally new ultrasound technologies also are emerging and offer exciting promise for making significant improvements in the clinical imaging of disease. These emerging methods include spectrum analysis, elasticity imaging, contrast-agent methods, and advanced flow detection and measurement techniques. Each provides independent information and, used alone, each can provide powerful new imaging capabilities; combined with each other, their capabilities may be even greater in many applications; and all in principle can be used in concert with other imaging modalities to offer the possibility of further improvements in disease detection, evaluation, and monitoring.
\end{abstract}

\section{Introduction}

Ultrasonic imaging has been a widely used and highly regarded method of visualizing anatomic structures for over 30 years. Two types of conventional ultrasonic imaging are commonly employed: grey-scale B-mode imaging and Doppler imaging.

Grey-scale B-mode images generated by conventional pulse-echo ultrasound display echo amplitude as a function of position in a scan plane. Echosignal amplitude is determined by the size, concentration, and relative acoustic impedance of weakly scattering constituents of tissue, such as blood vessels, connective-tissue strands, and glandular ducts and acini [1-3]. Smaller scatterer sizes, lower scatterer concentrations, and lesser acoustic-impedance differences between scatterers and their medium reduce echo-signal amplitude. Attenuation (due to absorption

${ }^{*}$ Corresponding author: Ernest J. Feleppa, Ph.D., Biomedical Engineering Laboratories, Riverside Research Institute, 156 William Street, New York, NY 10038-2609, USA. Tel: +1 212502 1775; Fax: +1 212502 1729; E-mail: feleppa@rrinyc.org. and scattering) also affects echo amplitude, and refraction (due to non-uniform propagation velocities) alters ultrasound beam properties and distorts image geometries and relative echo amplitudes. Nevertheless, conventional B-mode ultrasound imaging has proven to be extremely useful in detecting and diagnosing disease, guiding biopsies, planning therapy, and monitoring changes in tissue, e.g. those resulting from treatment.

Doppler images sense the shift in the frequency of echo signals relative to transmitted signals and portray motion, typically blood flow, in color. ColorDoppler imaging uses blue and red encoding, and power-Doppler imaging typically uses orange. (In color Doppler, red usually indicates motion toward the transducer and blue indicates motion away from the transducer. In power Doppler, the intensity of the orange color indicates the amplitude of echo signals from moving scatterers.) Such images often are supplemented with graphical displays of Doppler-signal spectra and acoustic outputs for aurally assessing Doppler information. 
While B-mode and Doppler methods of ultrasonic imaging are used widely in medicine and provide clinically important information, emerging methods of ultrasonic imaging potentially offer considerable additional information that may prove to be of great value as markers of disease. This article summarizes several such emerging methods with an emphasis on (1) spectrum analysis for assessing tissue micro-architecture and, hence, characterizing tissue types and properties; (2) elastography for sensing variations in tissue stiffness; (3) contrast agents for sensing blood flow and perfusion; and (4) sophisticated correlation-based techniques for depicting low-velocity blood flow.

\section{Emerging ultrasound technologies}

The core emerging technologies discussed here include those based on parameters computed from power spectra, elastographic techniques, contrast agents, and advanced flow-measuring methods. Related emerging techniques are discussed in each of these four contexts.

\subsection{Spectrum-analysis techniques}

Spectrum analysis utilizes the power spectra of radiofrequency $(\mathrm{RF})$ echo signals acquired in an ultrasonic scan plane [1-3]. Figure 1 shows a PC-based system for acquiring echo-signal data. A Hitachi EUB 525 scanner with two intra-cavity probes (typically used transrectally for prostate examinations) is shown interfaced with a PC-based data-acquisition system. RF data obtained internally from the scanner are digitized within the PC and stored on the hard disk of the computer. Data acquisition is controlled using the keyboard and auxiliary monitor shown at the upper right of the figure.

Tissue spectra typically are computed by applying a set of windows to RF echo-signal data from tissue to define a region of interest (ROI) and to select signals within that region for analysis. The two most common types of ROIs are those that contain windows of uniform size along a specified trajectory with respect to the scan vector, e.g. a rectangular ROI in a linear scan or a curved, constant-length ROI in a sector scan, and those that contain small, fixed-length windows that "slide" at specified range increments along each scan vector within an ROI of arbitrary shape, e.g. a 64-sample window stepping in eight-sample increments along every scan vector within the boundaries of a user-specified lesion. Figure 2 shows examples of both ROI types.
Figure 2(A) illustrates a constant-length ROI consisting of 58,110-sample windows; this ROI matches the location of a tissue biopsy in a prostate gland. The grey-scale B-mode image of Fig. 2(A) was computer generated from the digitized RF echo signals. Figure 2(B) shows a calibrated log power spectrum computed from the ROI of Fig. 2(A). The means of computing the spectrum is described below. Figures 2(C) and (D) illustrate ROIs of arbitrary shape filled with a large number of small windows; these ROIs match the apparent boundary of cancerous (Fig. 2(C)) and non-cancerous (Fig. 2(D)) breast lesions. Typically, "soft" windows such as cosine-squared, Hamming, or Hanning functions are used to strike a balance between spectral resolution and stability versus artifacts resulting from abrupt signal-amplitude changes caused by sharply opening and closing windows, e.g. as can occur if a rectangular window is used.

Once RF data are selected by a window, a Fourier transform is applied to the windowed signals, and the magnitude of the transform is squared. The result is the power spectrum of the windowed RF signals. In most applications, this result is expressed in decibel units as 10 times the log of the squared magnitude of the Fourier transform, and it is termed the "log power spectrum". In many applications, an additional "normalization" or "calibration" step is performed in which the log power spectrum of a perfect reflector, e.g. the surface of a glass or stainless steel block, is subtracted from the log power spectrum of tissue echo signals; calibration corrects for the transfer function of the system being used. In principle, this calibrated log power spectrum allows computing system-independent spectral parameter values and, furthermore, allows estimating scatterer properties such as size and relative acoustic impedance combined with concentration [1-3].

The first step in estimating tissue-scatterer properties is to calculate spectral-parameter values by performing a linear-regression analysis of the computed log power spectrum over the useable bandwidth of the system (i.e., the noise-limited bandwidth). Linear regression provides a "straight-line" approximation to the spectrum in which the spectral-parameter values (i.e., the slope, intercept, and midband values of the linear-regression line) provide a simple means of representing the spectrum. An example is illustrated in Fig. 2(B). These parameter values then can be related to scatterer properties. (Note that only two of these spectral parameters are mathematically independent. The relationship among them is $y=y_{0}+m f$ where $y$ is the amplitude value of the line, $y_{0}$ is the intercept, $m$ is the slope, and 


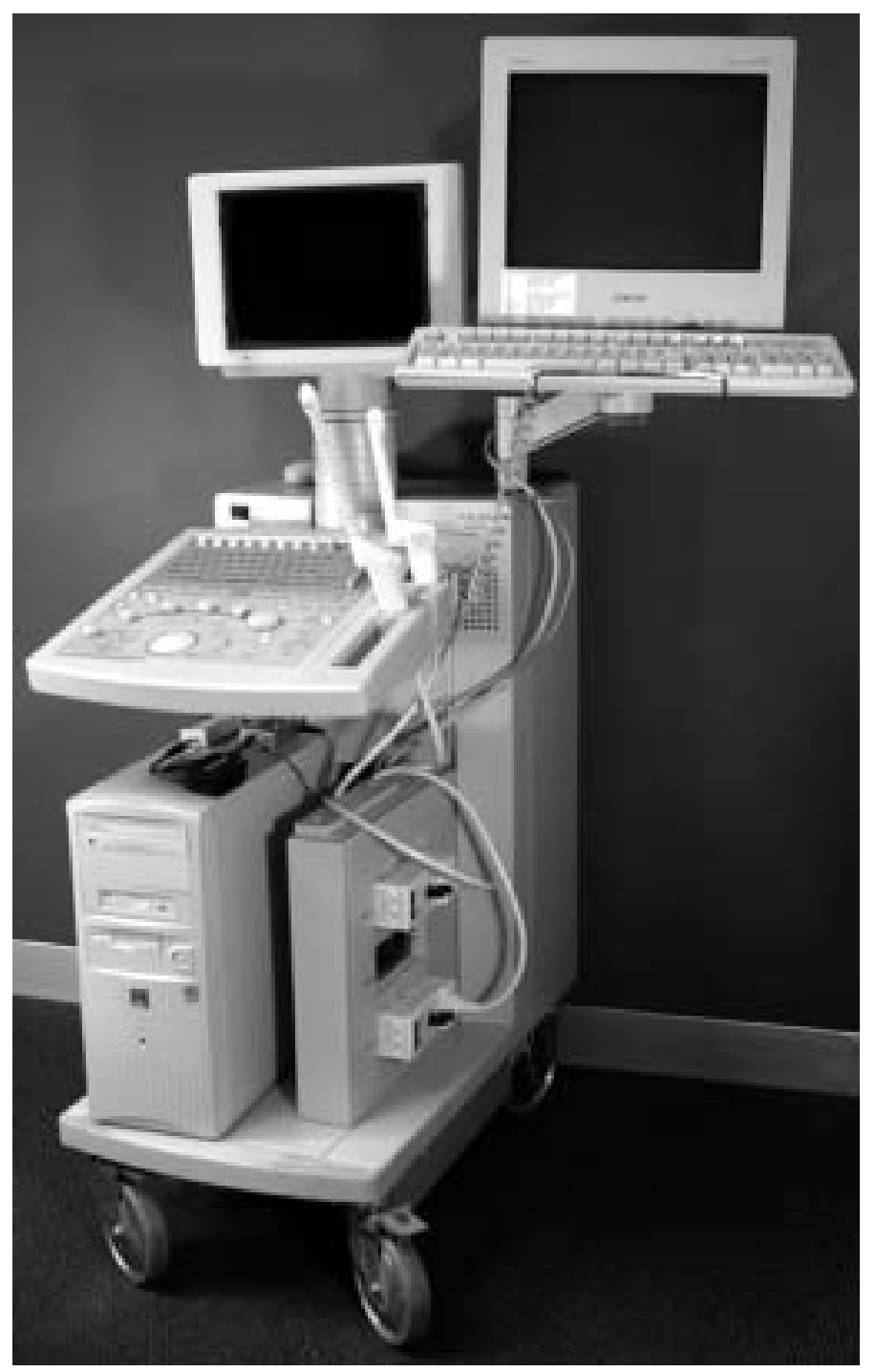

Fig. 1. Clinical scanner configured for data acquisition. A Hitachi EUB 525 instrument provides RF data to the PC computer system on its lower shelf (in the lower left corner of the figure). The keyboard and larger monitor (in the upper right corner of the figure) provide the user interface for the data-acquisition computer. RF signals are acquired from the scanner after summation of the individual array-element signals but prior to non-linear amplification or other processing.

$f$ is the frequency. If $f=f_{c}$, the center frequency, then $y=y_{c}$, the midband value.) As shown by Lizzi et al., if attenuation is taken into account, spectral slope provides a measure of scatterer size [1-3]. Up to the Rayleigh limit, scatterer slope value increases as scatterer size decreases. In contrast, the spectral intercept decreases with decreases in scatterer size, concentration, and relative acoustic impedance (i.e., the acoustic impedance of the scatterers relative to the acoustic impedance of the scatterers' medium). If scatterer size is known from the slope value, then the product of scatterer concentration and squared relative acous- tic impedance can be computed from the intercept value. Scatterer-property estimates depend upon the gate function (window) used to select RF signals and upon the two-way-directivity function (profile properties) of the ultrasound beam. See Lizzi et al for an in-depth discussion of the underlying theoretical framework [13].

\subsubsection{Spectral-parameter imaging}

Spectral-parameter imaging computes spectral-parameter values at every window location within a specified ROI and displays the parameter value at each pixel 

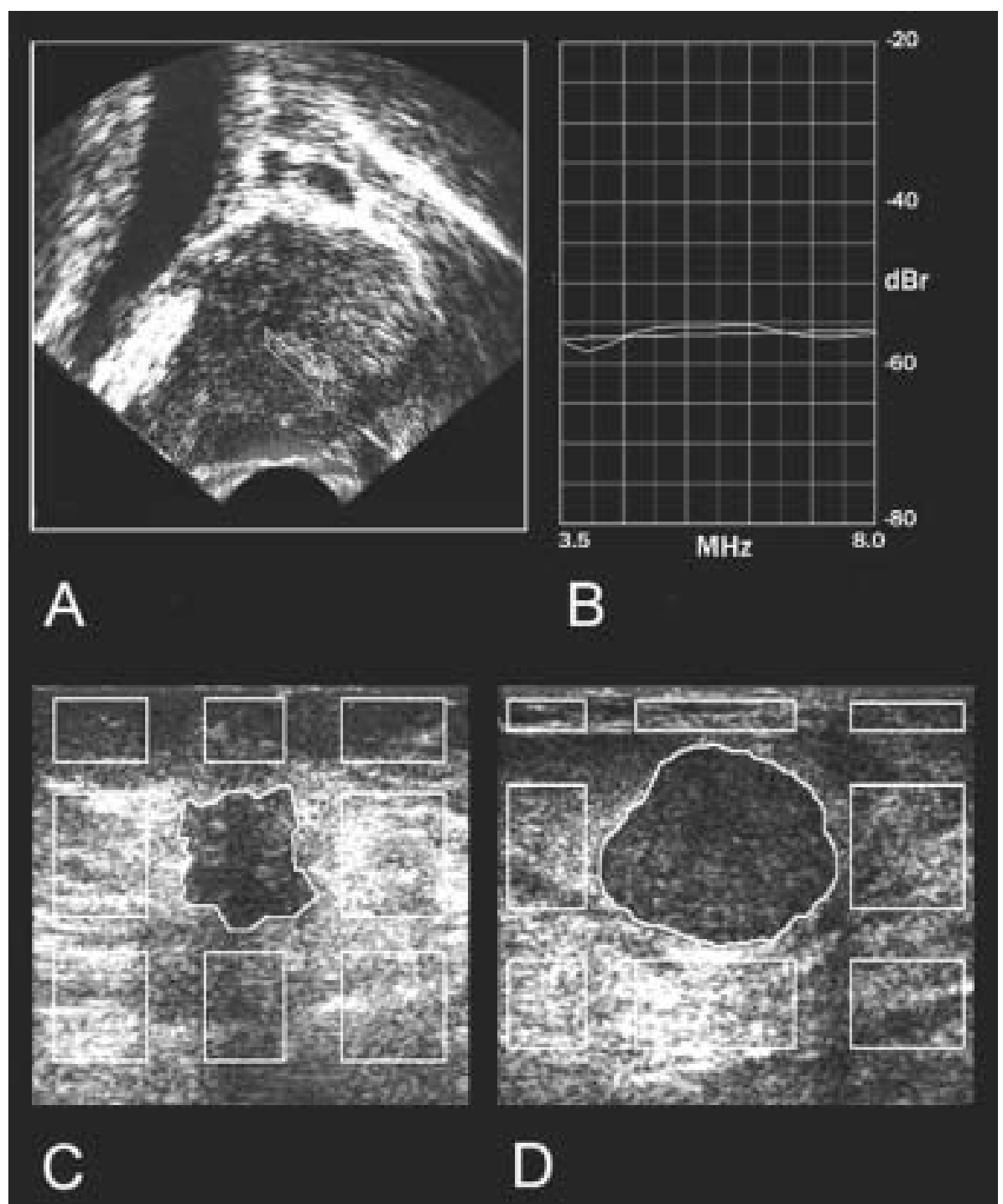

Fig. 2. ROIs and a spectrum for prostate and breast studies. A: Rectangular ROI in a longitudinal prostate scan. The ROI is placed where the biopsy needle sampled tissue immediately after scan RF data were acquired. The prostate is the central circular hypoechogenic region surrounding the ROI, with the seminal vesicles extending to the lower left of the scan. The distance from the inner to the outer arcs of the scan is $3.6 \mathrm{~cm}$. B: Prostate spectrum. The spectrum illustrates the useable bandwidth of prostate RF data acquired with typical prostate scanners; i.e., from 3.5 to $8.0 \mathrm{MHz}$ in this example. The spectrum is characterized by the slope, intercept, and midband values of its linear-regression approximation. C: An irregular, arbitrary-shape ROI used to compute spectral parameters for a cancerous breast lesion. (Rectangular ROIs are used to characterize disease-free tissue.) D: An irregular, arbitrary-shape ROI used to compute spectral parameters for a breast fibroadenoma. (Figures 2(A) and (B) are reproduced by permission of Ultrasonic Imaging.)

location as a grey-scale intensity. In practice, sliding windows are separated in range by distances smaller than half the window length, and grey-scale intensity at each pixel is computed as the average of the parameter values computed at several adjacent window positions. (This average includes windows on neighboring scan vectors as well as along the scan lines centered on the pixel.) The number of windows in the average is a trade-off in which a larger number of windows degrades spatial resolution but improves the statistical validity of the spectral parameter estimates [4]. Figure 3 shows examples of B-mode, slope, intercept, and midband images of a prostate gland in a transverse scan. The slope image is quite "noisy" compared to the midband and intercept image. The most stable image is the midband image, which is equivalent to the integrated-backscatter 


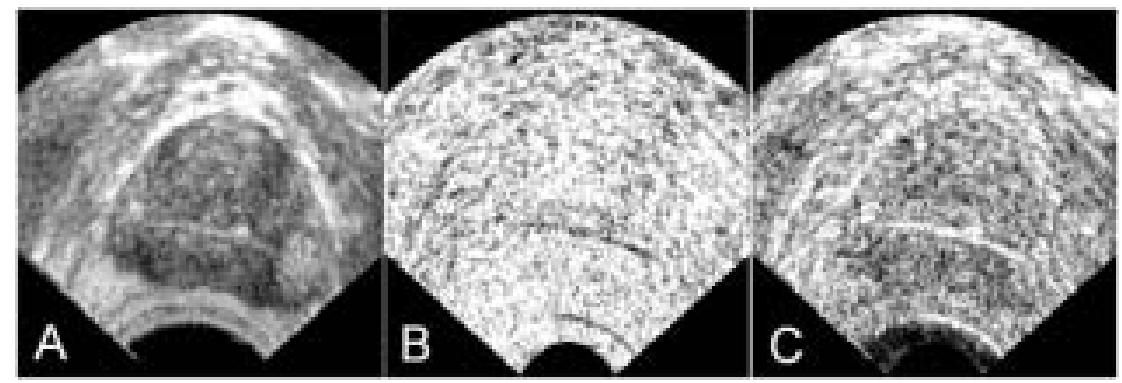

Fig. 3. Parameter images of the prostate in a transverse scan. The prostate is the heart-shaped region in the center of each image. The rectal wall is at the apex of the sector. A: Midband image. B: Slope image. C: Intercept image. Note that the slope image is very "noisy" and would benefit from a greater RF-signal bandwidth.

images of Miller et al. [5]. In fact, the so-called Bmode image of Fig. 3 is computer generated from the digitized RF echo signals. The B-mode and midband images are quite similar; however, the midband image improves the signal relative to speckle and provides a quantitative, system-independent image at the cost of some spatial resolution (which results from the averaging that occurs in generating pixel values from midband-parameter values at each window location).

Parameter values computed over a scan plane can be imaged themselves or can be used to generate other image types, such as scatterer-property or tissue-type images. The latter are discussed below. A notable example is the midband value expressed as integrated backscatter by Miller and co-workers, which has proven to be a very useful indicator of myocardial status in numerous studies by these investigators [6-8]. Miller and co-workers have been applying parametric imaging methods to characterization of cardiac tissue, particularly for the purpose of detecting and evaluating ischemia in the myocardium. In Miller's studies, the temporal and angle-dependent characteristics of attenuation-corrected integrated backscatter (which is equivalent mathematically to the midband parameter described above) have proven to be a powerful tool in assessing the mechanical properties of the myocardium.

The limitations of these methods are those imposed by the physics of coherent acoustical imaging and the statistics of estimating spectral parameters. These physical limitations arise from well-known propagation and beam phenomena, e.g. limits on lateral resolution associated with transducer aperture and frequency and limits on axial resolution imposed by bandwidth [9]. Additional limitations result from phase aberrations caused by inhomogeneities in propagation velocity, near-field artifacts, diffraction effects and beam broadening, band-limiting noise arising from the system and the environment, motion due to respiration, heart contractions, arterial pulses, and the like. Speckle is a well-known phenomenon of coherent-imaging systems; speckle overlays image detail with randomly produced small-scale interference patterns and it masks small structures. The statistics of estimating spectral parameters are discussed in detail by Lizzi et al. [4].

\subsubsection{Scatterer-property imaging}

Generating scatterer-property images from parameter-value images requires only a simple algorithm that takes into account the window and transducer properties along with the system bandwidth [1-3]. Once parameter values are computed at each pixel position, the algorithm converts the parameter values to values representing estimates of size, $d$, and the product, $C Q^{2}$, of concentration, $C$, and squared relative acoustic impedance, $Q$, which sometimes is termed "acoustic concentration". Intraocular examples from the work of Silverman, Coleman, Lizzi and others are shown in Fig. 4. Figure 4(A) shows a scatterer-size (d) image of an intraocular melanoma; Fig. 4(B) shows an acoustic-concentration $\left(C Q^{2}\right)$ image of ciliary muscles that depicts changes that occur during ocular accommodation [10,11]. Furthermore, Silverman and co-workers have shown that parameter-value changes follow changes in tissue, specifically in choroidal malignant melanomas, that result from radiation therapy [12]. Oelze et al. have investigated parameter and tissue-property techniques in several phantom and animal studies, including characterization of mammary tumors in experimental rats $[13,14]$.

Insana et al. have developed an analogous method of estimating scatterer size [15-17]. This method relates the frequency-dependent backscatter coefficient to $f$, $d, C Q^{2}$, and a form factor, $F(f, d)$. If scatterers are not spherical, but are symmetric non-spherical entities, then the form factor becomes a function of scanning an- 


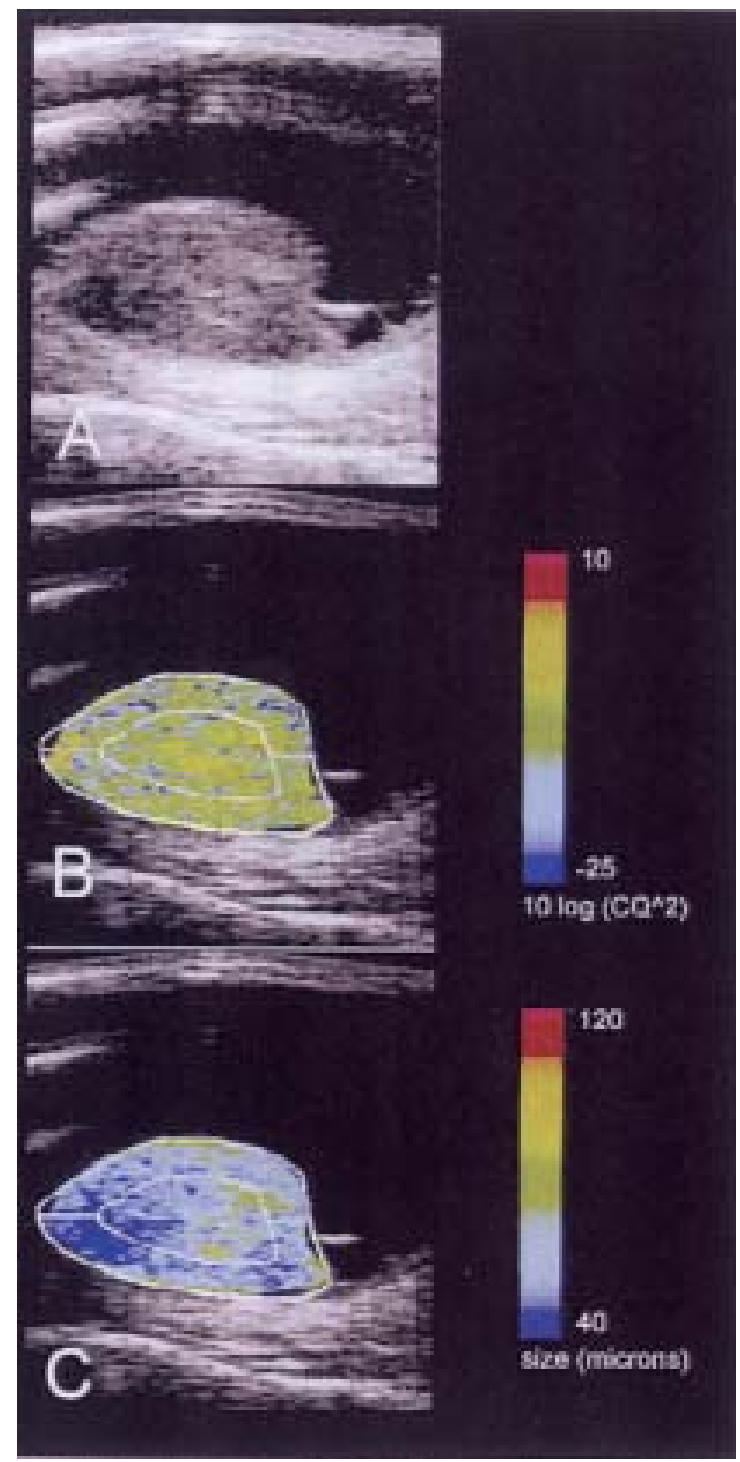

Fig. 4. Scatterer-property images. B-mode and scatterer-property images for a posterior, intra-ocular, choroidal, malignant melanoma. The single-element, mechanical, sector-scanning transducer is above the eye in these images. (The eye appears flattened because the original 30-degree sector geometry is presented in a rectilinear format.) A: B-mode image showing the tumor extending into the vitreous humor from its base in the choroid. B: $C Q^{2}$ image showing a range of values for $10 \log C Q^{2}$ extending from -25 to $+10 \mathrm{~dB}$. C: Scatterer-size image showing scatterers from as small as $40 \mu \mathrm{m}$ to as large as approximately $80 \mu \mathrm{m}$. (Printed with the permission of Ronald Silverman, Department of Ophthalmology, Weill Medical College of Cornell University.)

gle. In this approach, sizes are estimated by comparing modeled form factors with form factors derived from measured backscatter coefficients. Insana and his collaborators have successfully applied this method to esti- mating the sizes of kidney structures such as glomeruli, tubules, and arterioles [17].

The limitations of scatterer-property imaging derive from those of acoustical imaging and parameter imaging. The statistical aspects are described by Lizzi et al. [4].

\subsubsection{Tissue-type imaging}

Tissue-type imaging requires a database containing spectral-parameter values for the types of tissues that are of interest, e.g. primary choroidal melanomas vs. metastatic choroidal lesions in the eye or adenocarcinomas vs. non-cancerous tissues in the prostate $[12,18-$ 27]. A classification tool, such as linear-discriminant analysis, nearest-neighbor analysis, or neural-network analysis, is applied to the data to determine what parameter values are associated with each tissue type of interest. In some cases, such as the prostate, including a clinical variable, such as the blood level of prostate-specific antigen (PSA) can improve classification [23]. Once the optimal classifier is established, that classifier can be used to translate parameter values at each pixel location into a score to indicate tissue type, e.g. a high score value to indicate a high likelihood of cancer in a prostate image. The score can be depicted in grey-scale or using colors to show values within pre-specified score ranges, e.g. red to show very high likelihood of cancer and orange to show a moderate likelihood. Figure 5 shows examples of tissue-type images derived from a transverse scan of the prostate. Figure 5(A) shows a grey-scale cancerlikelihood image, and Fig. 5(B) shows a color-encoded image in which red and orange depict the two highest likelihoods of cancer against a midband-value image background. The whole-mount histologic section of the prostatectomy specimen is shown in Fig. 5(C). The patient's prostate was scanned in a transverse mode immediately prior to prostatectomy surgery using 5$\mathrm{mm}$ spacing between scan planes. The pathologist sectioned the excised prostate using 3-mm spacings between sectioning planes and then demarcated cancers and pre-cancerous lesions in each whole-mount section. Figure 5 shows planes that approximately match; i.e., that have the greatest lateral dimension compared to others in the set. The demarcated region at the top of Fig. 5(C) is a 12-mm anterior tumor, which matches the bright anterior region at the top of Fig. 5(A) and the large red and orange region at the top of Fig. 5(B). Images from adjacent planes are assembled into the 3-D surface rendering of Fig. 6. Red depicts the surfaces of high-suspicion regions within the light-grey prostate 


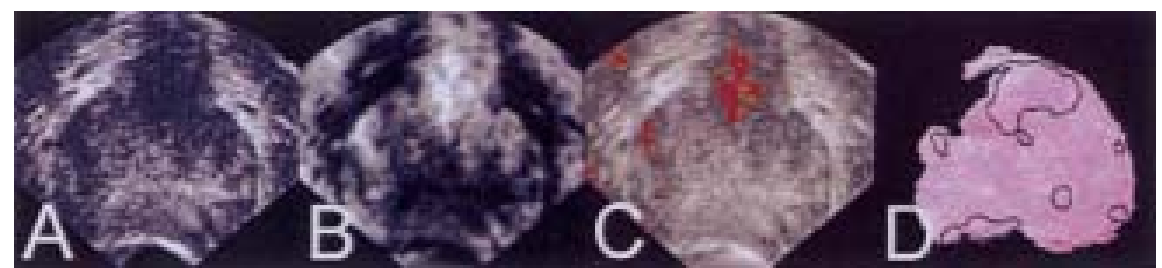

Fig. 5. Ultrasonic tissue-type and histologic images of a prostate with an unsuspected anterior tumor. Ultrasonic RF data were acquired immediately prior to prostatectomy for comparison with subsequently obtained whole-mount histology. A: B-mode image showing artifact-like shadowing that masked the presence of the tumor. B: Grey-scale tissue-type image in which brighter pixels denote higher likelihood of cancer. The bright anterior region is consistent with the subsequent histological determination that an anterior tumor was present. C: Color-encoded tissue-type image depicting the highest levels of likelihood for cancer as red and orange superimposed on a midband image. D: Histological cross-section (in a plane closely matching the ultrasound plane of A, B, and C) that shows a 12-mm anterior tumor along with smaller tumor foci and a posterior pre-cancerous condition. (Note that Fig. 5 views the gland from the apex.) (Reproduced by permission of Ultrasonic Imaging.)

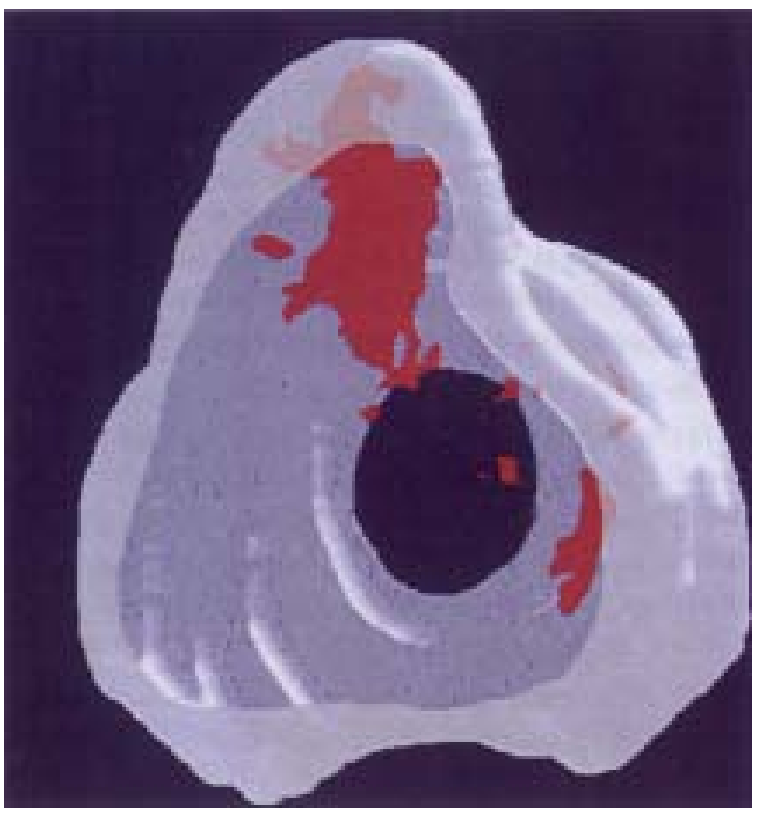

Fig. 6. 3-D rendering of the prostate gland of Fig. 5. The 12-mm anterior tumor of Fig. 5 is clearly depicted, along with several smaller foci of cancer. (Note that Fig. 6 views the gland from the base; i.e., the perspective taken in Fig. 6 is the reverse of the perspective taken in Fig. 5). (Reproduced by permission of Ultrasonic Imaging.)

capsule. (The capsule was demarcated manually using computer-generated B-mode images derived from the original RF data.)

Although the limits of parameter imaging extend to tissue-type imaging, the more constraining limitations are those associated with the tissue classifier, and these limits are best described in terms of classification sensitivity, specificity, positive or negative predictive values, ROC curves, and the like, in each particular application [28]. In most cases, image resolution is not likely to be the driving issue in this sort of imaging compared to the reliability of tissue-type or tissue-change deter- mination, as is addressed further in the discussion of applications below.

\subsection{Elastography techniques}

Tissue elasticity depends on molecular building blocks and their structural organization, which can be altered by pathological conditions such as cancer [29]. Variations in tissue elasticity can serve as markers of disease, e.g. cancers, which tend to be stiffer than normal tissues [30]. Physicians routinely use palpation to detect cancers in the breast and other organs such as the prostate. However, palpation is limited to shallow lesions or cases where elasticity differences are large. Furthermore, palpation does not provide quantitative information, and its utility depends on physician skill and experience. Ultrasound-based elasticity imaging methods have been developed to overcome these limitations and to provide a means of evaluating tissues based on local variations in elasticity. While conventional B-mode ultrasonograms convey information regarding acoustic-scattering properties, elasticity images convey information regarding tissue elasticity, primarily Young's modulus. Elasticity and sonographic parameters usually are unrelated so that elastograms and ultrasonograms contain different types of information. Elasticity imaging methods enjoy an inherently large dynamic range; differences in tissue elasticity can be a few orders of magnitude in the presence of abnormalities [30], as opposed to only a few percent typical of other imaging modalities. Various groups have reported on the stiffness values of biological tissues. This sensitivity to tissue type has motivated widespread investigations of elasticity imaging. Ultrasonic elasticity-imaging methods employ two different approaches: (1) imaging of tissue response due to quasi-static loading or compression [31,32], and (2) 
imaging of tissue response due to dynamic loading or vibration, which typically uses Doppler-based methods to sense local vibration [33-36].

The compression-based approach, usually termed elastography, produces images that quantitatively depict local tissue deformation. To date, elastography has been tested mostly under in vitro conditions, has demonstrated great potential for a number of clinical applications; initial clinical studies for breast-cancer detection are very encouraging [37]. Figure 7 illustrates the potential of elastography in imaging cancers of the breast. Figure 7(A) shows a conventional Bmode breast scan obtained with a linear-array probe positioned at the top to the scan; Fig. 7(B) shows the corresponding elastogram. Data were acquired using a Diasonics Spectra scanner with a 7.5-MHz center frequency linear-array probe. The conventional B-mode image hints at the presence of a $1.5-\mathrm{cm}$ ductal carcinoma as the hypoechogenic region near the top of the image and slightly to the left of center; the presence of the lesion is reinforced by the shadowing that is distal to it (with respect to the transducer). The conventional image shows the lesion to have a "U" or "V" shape. The elastogram more clearly displays the lesion as a dark, relatively low-strain (high-stiffness) "V" at the top of the image and slightly to the left of center. (Other low-strain elements in this image appear to be Cooper's ligaments or other normal fibrous components of the breast.) The elastographic depiction shows slightly larger lesion dimensions than does the B-mode depiction, which is characteristic of a desmoplastic reaction that stiffens the tissue surrounding malignant lesions.

Vibration-based methods include sonoelasticity imaging. Sonoelastograms show the vibration amplitude pattern of shear waves that result from external vibration using color imaging (similar to color Doppler displays) on a conventional B-mode grey-scale image. A theory of sonoelasticity imaging was developed and in vitro results on excised human prostate were promising. This method recently has been extended to 3-D to enable visualization of tumor volume [38]. Sonoelasticity images typically are non-quantitative, and resolution may be relatively poor. However, vibration can be applied more easily than quasi-static loading and is more amenable to freehand application.

\subsubsection{Elastography}

This section briefly describes elastography, which is discussed in detail in numerous publications [31,32,3641]. Compression typically is applied through the linear array probe used for imaging; in some cases, the surface of the array may be expanded by using an attached flat plate. Compression occurs between the fixture that applies the compressive load to the tissue and some rigid structure behind the tissue, e.g. the rib cage in breast studies or the pubic arch of the pelvis in transrectal prostate studies. Currently, most studies employ some sort of computer-controlled application of the compression fixture. The fixture is applied to the surface of the tissue being interrogated and is advanced to deform the tissue. Compression is the term loosely used to refer to the response of the tissue to this deformation. Although application of the deformation force may force blood from vessels in the loaded tissue, in fact tissue is not literally compressed. Instead, scatterers move within the deformed tissue and the tracking of this movement is the basis of strain estimation underlying elastography.

Strain, the quantitative descriptor of internal tissue deformation, is defined as $\varepsilon=\Delta L / L$, where $\Delta L$ is the change in initial length $L$. The procedure to generate conventional elastograms consists of the following steps:

(1) A frame of RF echo-signal data is acquired from tissue prior to tissue compression.

(2) Tissue is compressed along the axis of the transducer by the computer-controlled fixture.

(3) A second frame of RF data is acquired after tissue compression.

(4) Acquired frames are analyzed to estimate the induced tissue displacement using cross-correlation analysis of pre- and post-compression RF signals. The local gradient of displacement provides estimates of local strain, $\hat{\varepsilon}=\Delta \tau / \Delta t$, where $\Delta \tau$ is the estimated difference between the compression-caused displacements computed at different window positions, and $\Delta t$ is the difference in successive window positions. Here, $\Delta \tau$ and $\Delta t$ are expressed in units of time; however, if acoustic propagation velocity is known and is uniform, they can be expressed in units of distance.

Echoes along each scan line are segmented into overlapping temporal windows, and strain is estimated throughout a tissue cross-section to form an elastogram.

When tissue is compressed, echo signals undergo not only a delay, but also experience a change in their shape, which causes a loss of correlation between pre- and post-compression echo signals. Because tissue is virtually incompressible (Poisson's ratio, $\nu \approx 0.5$ ), deforming it may cause non-axial motions and out-of-plane 


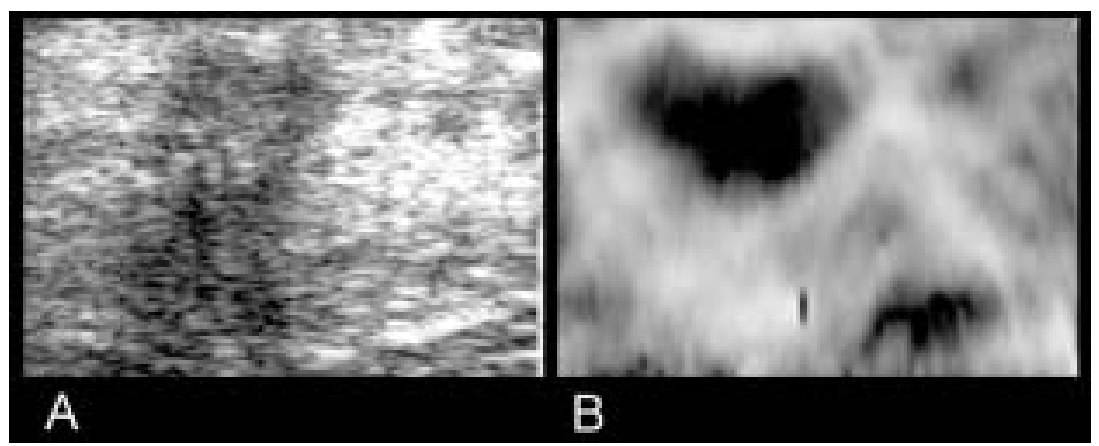

Fig. 7. Conventional sonogram and elastogram of a 1.5- $\mathrm{cm}$ ductal carcinoma of the breast. A: B-mode ultrasound image of a breast lesion. The lesion is the U- or V-shaped hypoechoic region at the top of the scan with posterior shadowing extending downward (away from the transducer). B: Elastogram of the lesion. The lesion is seen clearly as the V-shaped dark region at the top of the scan; darker pixels in an elastogram indicate lower strain (i.e., greater stiffness). The lesion appears slightly larger in the elastogram than in the sonogram, which is characteristic of desmoplastic malignant lesions. (Printed with the permission of Brian Garra, Department of Radiology, University of Vermont.)

displacements that introduce additional decorrelation. These effects increase as strain increases. Therefore, small tissue strains generally are preferred in elastography.

Use of small compression steps limits non-axial and out-of-plane motion. However, at times, either larger motion is desired or limiting it may not be possible, e.g. if compression is applied manually. Approaches to estimate and correct for non-axial tissue motion have been developed [42-44]. Deformable-mesh algorithms recently have been applied to complex, ultrasonic-motion analysis for tracking complex displacements; these algorithms may facilitate handheld applications $[45,46]$. When linear arrays are used for compression, azimuthal motion can be reduced by mechanically confining the tissue. Due to the essentially incompressible nature of tissue, restricting azimuthal displacement increases the elevational motion. However, the ultrasonic beam is broader in the elevational direction; thus, the effect of elevational displacement is less pronounced. Under certain assumptions, incompressibility processing could be used to compute lateral strains from axial strains. However, this method requires accurately estimating the zero-lateral-displacement contour, and it assumes a Poisson's ratio of 0.5 , which may not always be valid.

The performance of elastography is limited by mechanics as well as ultrasound physics. Reduction of the contrast of a target inside a uniform background is characterized by Contrast-TransferEfficiency (CTE) [47,48], defined as the ratio of the observed (axial) strain contrast measured from the strain elastogram and the underlying true modulus contrast. Stiff low-contrast stiffer lesions have high CTE (close to $0 \mathrm{~dB}$ ), thus the strain map is representative of the modu- lus map. Stiff inclusions have been shown to have better CTE than do softer lesions; a surrounded incompressible soft inclusion cannot deform under pressure as it might otherwise do without constraints. While CTE limits actual strain contrast, visualization of this strain is limited by noise and ultrasound physics. Varghese and Ophir developed a theoretical framework, termed Strain Filter, to characterize the maximum attainable elastographic signal-to-noise ratio vs. local strain as a function of system as well as processing parameters, e.g. center frequency, bandwidth, data window size, window shift, etc. [49]. Strain Filter analysis in combination with CTE can predict the elastographic contrastto-noise ratio performance (CNRe) [50]. Righetti et al. have shown that the ultimate resolution of elastography is of the same order as the B-mode resolution [51]. However, practical limits of elastographic resolution were shown to be a linear combination of data window size and window overlap, with window overlap having a larger weight [52]. Elastographic phantom development is very important in characterization studies, and there have been considerable efforts in this area [33,5355]. Commercial manufacturers such as CIRS, Inc. (Norfolk, VA) also provide elasticity phantoms to researchers.

Like any imaging modality, elastography presents numerous artifacts. While these are well understood in B-mode imaging, considerable effort is being directed toward understanding and minimizing elastography artifacts. Mechanical artifacts arise from macroscopic mechanical properties of tissue, e.g. stress concentrations. Acoustic artifacts arise from beam profiles, reverberations, phase aberrations, and the like $[41,56]$. Signal-processing artifacts, such as "zebras" and "worms", arise from sampling and analysis 
errors [40,57-59]. Numerous studies are underway to develop methods of reducing or eliminating these artifacts in elastographic images and improving the "quality" of strain estimation [52-78].

\subsubsection{Recent developments}

Radiation pressure from an ultrasound transducer can be used to compress tissue. Nightingale et al. showed the feasibility of using the same diagnostic transducer for both pushing and imaging the breast in vivo [79]. Lizzi et al. showed the feasibility of monitoring thermal lesions created by high intensity focused ultrasound (HIFU) [80]. Viola et al. used radiationforce elastography (also called "ARFI", Acoustic Radiation Force Impulse imaging) to analyze deep venous thrombosis (DVT) [81]. Fatemi and Greenleaf developed vibro-acoustography, which uses the radiation force of two intersecting continuous ultrasound beams to remotely vibrate an object at an arbitrary low ("beat") frequency [82]. By measuring the acoustic emission field, one can obtain information about the mechanical parameters of the object. This method has shown promise in mapping elasticity variations, but the entire tissue sample must be scanned with the intersecting transducers, making the imaging process exceedingly slow.

Conventional elastography compresses tissue with a relatively large, computer-controlled mechanical fixture to limit deformations and minimize non-axial tissue motions and to reduce decorrelations between preand post-compression echo signals. Manual compression cannot reliably control transducer movement and typically results in motion with significant nonaxial components that cause rapid estimator degradation. Conventional processing methods cannot produce useful images using the resultant data. Therefore, methods that allow applications in handheld techniques would be very useful and recently have been investigated $[72,83,84]$. Although freehand implementation of elastography is a difficult problem, preliminary systems have been constructed and more sophisticated systems are expected to be constructed in the near future.

While tissue strain is not an intrinsic tissue property, Kallel et al. have shown that strain maps may be a reasonably good representation of underlying stiffness distribution for low modulus contrast in the presence of simplified boundary conditions [54].

\subsection{Contrast-agent techniques}

Clinical applications of ultrasonic contrast agents (UCAs) have been developed to increase the diagnostic capabilities of ultrasound imaging. Most UCAs are stabilized, microscopic, gas-filled, bubbles that are injected into the bloodstream. UCAs are capable of producing much stronger echo signals than are blood or tissue because of the substantial acoustic impedance difference between the gas inside the bubbles and the surrounding blood $[85,86]$.

The field of ultrasonic contrast imaging has developed rapidly in recent years because UCAs greatly can increase the ability of ultrasound to detect and image slow blood flow in small, deep vessels that are important in early disease detection, diagnosis, and monitoring $[87,88]$. In cardiology, UCAs are used in a wide range of applications, including the identification of cardiac structures, evaluation of perfusion (such as the detection of inadequate myocardial perfusion), and quantification of cardiac output $[60,89,90]$. Contrastassisted imaging has proven useful for characterizing liver lesions [61,91], detecting breast tumors [92-95], and detecting prostate cancer [96-99]. UCAs also offer potential for evaluating tumor-induced angiogenesis by providing an enhanced capability for measuring tumor flow and vascular volume. UCA imaging makes characterizing the structural and functional features of tumor vascularity possible from the earliest stages of tumor development [92].

Micro-bubble UCAs interact efficiently with diagnostic ultrasound, can exhibit linear and nonlinear behavior that generates harmonic and sub-harmonic signals $[100,101]$, and can be destroyed by ultrasound pulses [102,103]. While backscatter enhancement from contrast agents has proven useful in clinical applications, new techniques are being developed continuously by utilizing specific properties of contrast agents, based on the physical ultrasound-contrast agent interaction [104,105]. These new methods and techniques are developed to help flow detection and imaging [106] and to extract quantitative information [107] for both morphological and functional studies. There also have been studies to explore the feasibility of using contrast agents for very-high-frequency ultrasound (VHFU) applications [107]. In addition, development of organspecific agents and targeted agents may provide ultrasonic imaging enhancement of targeted tissue structures and open new routes for therapy [108,109].

UCA imaging techniques and new developments in UCAs are summarized below in the following three categories: basic UCA-assisted imaging, functional flow imaging, and targeting. 


\subsubsection{UCA-assisted imaging}

UCA-assisted imaging is summarized in terms of: basic contrast enhancement, non-linear techniques, and transient-imaging methods.

\subsubsection{Contrast enhancement}

Numerous studies have demonstrated the benefit of backscatter enhancement achieved by introducing contrast agents during ultrasound examinations [110,111]. These studies have shown that clinical application of UCAs improves fundamental B-mode image clarity and increases Doppler-signal amplitude. By increasing the effective reflectivity of the blood, UCAs enable better delineation of tumor boundaries, better detection of endo-cardial borders for assessment of wall-motion abnormities, and better detection of blood flow in small, deep vessels [90,110,112].

\subsubsection{Non-linear imaging}

Non-linear imaging techniques have been developed to exploit the strong non-linear properties of contrast agents [113]. Techniques such as harmonic, subharmonic, and pulse-inversion imaging exploit these characteristics of UCAs but require relatively stable contrast agent populations for optimal performance.

Second-harmonic B-mode imaging is a method where the ultrasound system generates images from backscattered signals at the harmonic frequency of the transmitted fundamental frequency $[114,115]$. Because UCAs injected into the bloodstream exhibit stronger non-linearity than does surrounding tissue, harmonic signals arise primarily from the UCAs in the blood. Thus, B-mode images generated from harmonic echo signals preferentially display vascular volumes and perfused tissues.

Sub-harmonic imaging has been proposed to generate images based on B-mode presentations derived from the backscattered sub-harmonic signals of the transmitted frequency signal $[101,116,117]$. Because subharmonic signal generation is minimal in tissue, subharmonic imaging has the potential for better differentiation of blood and tissue; yet further development of transducers and imaging strategies will be essential for the successful application of sub-harmonic imaging [118].

Pulse-inversion imaging uses a sequence of two phase-inverted pulses transmitted into tissue; then the sum of the two echo signals is processed for imaging [119-122]. For a linear medium, the sum of the two responses is zero because the second response is the inverted replica of the first response. However, the sum of the two responses for a non-linear medium is not zero because of waveform deformation resulting from propagation through the non-linear medium. The amplitude of the sum depends on the degree of non-linearity of the medium and, if system bandwidth is sufficient, it can include all of the even-harmonic components (not just the second harmonic signal) in the backscattered echo. Therefore, pulse-inversion methods can achieve better imaging resolution compared to simple harmonic imaging and harmonic power-Doppler imaging.

Power-Doppler imaging techniques display the power of the signal amplitude of Doppler signals to indicate flow existence rather than flow direction as is done in conventional Doppler techniques [115]. Because most Doppler techniques employ multiple pulses, they are susceptible to tissue motion. In some cases, tissue motion can generate Doppler signals that are comparable with or even overshadow contrast-agent signals. As in harmonic B-mode imaging, harmonic power-Doppler imaging displays the power of the second harmonic Doppler signals, which primarily are contributed by contrast agents and therefore can better differentiate flowing blood from moving tissue [118,119].

Other recent developments of clinical UCA imaging include power-pulse inversion (Philips Medical Systems, Bothell, WA), power modulation (Philips Medical Systems, Bothell, WA), and agent-detection imaging (Acuson Corporation, Mountain View, CA).

Power-pulse inversion (PPI) combines pulse-inversion harmonic methods with power-Doppler methods to further increase sensitivity to contrast agents. This new technology uses additional pulses to virtually eliminate the tissue signal from the UCA signal. Then contrast agents are displayed in color, while tissue is displayed in fine-resolution grey-scale. This new technology allows the display of minute amounts of contrast while simultaneously displaying a fine-resolution 2-D image in real time to allow the physician to clearly distinguish blood containing UCA from surrounding tissue.

Power modulation uses a multi-pulse technique in which the acoustic amplitude of two separate transmitted pulses is different: full and half amplitude. This amplitude change induces changes in the response of the contrast agent. The received echoes from the half-amplitude transmitted pulse are amplified twice as much as are the full-amplitude pulses and, subsequently, are subtracted from the full-amplitude echoes. This procedure removes most of the linear responses at the fundamental frequency, and the remaining echoes are dominated by the non-linear signals from the UCAs. Power modulation also is used with a low- 
frequency wide-band transducer. The low-frequency transducer increases the penetration depth and depth of field, and transmits the ultrasound energy more uniformly throughout the image. The combination of power modulation and a wide-band transducer allows ultraharmonic imaging, which provides better elimination of tissue artifacts and therefore results in an increased contrast between UCA and tissue-echo signals.

The agent-detection imaging mode (Cadence Contrast Agent Imaging) offered by Acuson (Acuson Corporation, Mountain View, CA) provides progressive contrast-agent imaging through Power Contrast Imaging, Color Harmonic Imaging, and Low Mechanical Index Optimization.

\subsubsection{Transient imaging}

Micro-bubble UCAs can be destroyed by ultrasound to produce transient effects [102,103]. Transientimaging techniques such as flash-echo imaging, triggered imaging, and release-burst imaging employ transient-enhanced scattering from ultrasonic contrast agents for optimal imaging of contrast agents [118124].

Release-burst imaging is a novel contrast-imaging approach that exploits the transient characteristic of UCAs. It uses a combination of multiple, highfrequency, broadband, detection pulses and a separate release burst. The detection pulses survey the target before and after transient enhanced scattering that results from the release burst. The presence of the UCA is detected by correlating or subtracting the pre- and postburst signals. Because the time interval between the two detection pulses is minimal (approximately 200$400 \mu \mathrm{s}$, depending on the scan depth, size of the region, etc.), subtraction of the RF data can be performed in real-time and is less susceptible to data-acquisition instabilities.

Stimulated acoustic emission (SAE) generated by micro-bubble disruption has been used to image liver diseases [106]. At high acoustic pressures, e.g. typical color-Doppler levels, gas-filled UCAs can be destroyed to produce "stimulated acoustic emission", which produces strong, transient grey-scale enhancements or characteristic mosaic-like signals on colorDoppler imaging. Using SAE, stationary or slowmoving UCA bubbles can be detected within liver and spleen parenchyma.

\subsubsection{Functional imaging and flow quantification}

UCAs are contained entirely within the vascular space, travel at the same velocities as the blood, and can be relatively stable over a period of time suitable for ultrasonic examination. In addition to increasing Doppler-signal amplitudes for flow measurement, contrast agents are useful in other methods of flow measurement such as in indicator-dilution blood flow analysis [125]. More recently, destructive flow-estimation methods have been reported [126,127].

Following the indicator-dilution principle, timevideo intensity (indicator-dilution) curves from media with contrast agents are used to estimate flow rates [128]. Destructive methods ultrasonically manipulate UCA bubbles using various techniques, including radiation force [103]. Such time-domain techniques measure backscatter before and after an induced change in the flowing UCA population. The time curve of backscatter then is used to estimate local flow rate [129].

Tracking the passage of a bolus of UCA bubbles through a region of interest gives functional information on circulation. Clinical applications can be divided into those that interrogate the entire organ and those that study only one region, such as a tumor. By characterizing the transit time of inflow/outflow or monitoring "reperfusion", perfusion can be estimated from the time required for a region to refill with UCAs after they have been destroyed $[125,126]$.

\subsubsection{Targeting}

Contrast-assisted ultrasonic imaging is expanding beyond the assessment of micro-vascular perfusion because ultrasonic contrast agents often cannot specifically differentiate abnormal from normal tissues except by revealing altered blood-flow patterns. Development of site-specific UCAs potentially can provide more definitive information to differentiate healthy and pathological tissues by improving the capability to discriminate and localize various tissue pathologies or cell-surface markers [6,7]. The application of these agents includes diagnosis, therapy, biopsy, or resection. Site-specific UCAs have been used for imaging targeted tissue, including use as a marker for visualizing inflammation, thrombi, and angiogenesis and as an adjunct means of enhancing drug delivery and gene therapy [130-134].

\subsection{Advanced flow-assessment techniques}

Advanced techniques for sensing and depicting flow at extremely low velocities and in small vessels are being studied by a number of investigators [135139]. These techniques do not use Doppler methods, but instead use correlation methods to estimate the 
shift of echo signals in range as a function of time; they use high-frequency ultrasound (HFU) or veryhigh-frequency ultrasound (VHFU) with swept-mode (scanned beam) imaging to locate and measure vessels and with M-mode (fixed beam location) data acquisition to quantify flow [135-139]. These techniques ordinarily estimate flow by tracking feature movement in M-mode images, but they also can use color-flow displays to depict flow after aligning adjacent scan lines by removing motion artifacts and by applying a "Wall" filter (essentially a high-pass filter) to remove artifacts from stationary elements. The local phase of the filtered signal then can be color encoded and superimposed on the swept-mode image. Figure 8 shows examples of the swept-mode method applied to microperfusion in the rabbit eye, specifically, to the thermoregulatory mechanisms of the major arterial circle of the iris. Figures 8(A) and (B), respectively, show swept-mode and color-Doppler images of the arterial circle at $37^{\circ} \mathrm{C}$; Figs $8(\mathrm{C})$ and (D), respectively, show swept-mode and color-Doppler images at $4{ }^{\circ} \mathrm{C}$. (The eye of the anesthetized rabbit was proptosed and scanned in a temperature-controlled saline water bath.)

\section{Current and potential applications}

The emerging ultrasonic methods briefly summarized here have a broad range of applications in medicine and biological research. Clearly, tissue typing based on spectrum analysis, elastography, or targeted contrast agents has enormous potential benefit in detecting, evaluating, treating, and monitoring many types of disease lesions. Cancerous lesions, ischemic myocardium, and intra-vascular lesions associated with cardiac and cerebral ischemia are a few obvious examples. Although they now are emerging as exciting tools for detecting early markers of disease, many of these methods have been investigated for several years. Lizzi, Coleman, Silverman, and their co-workers have been developing and refining many of the spectrum-analysis approaches in the course of their foundation ophthalmic intraocular cancer studies that were initiated nearly three decades ago $[12,18-$ 21,140]. As described above and as illustrated in Figure 5, Feleppa and co-workers have, for over a decade, been investigating the use of spectrum-analysis methods and non-linear classifiers to enable imaging of cancerous lesions of the prostate for the purpose of guiding needle biopsies and for targeting and monitoring therapy [22-27]. Alam et al. have developed a

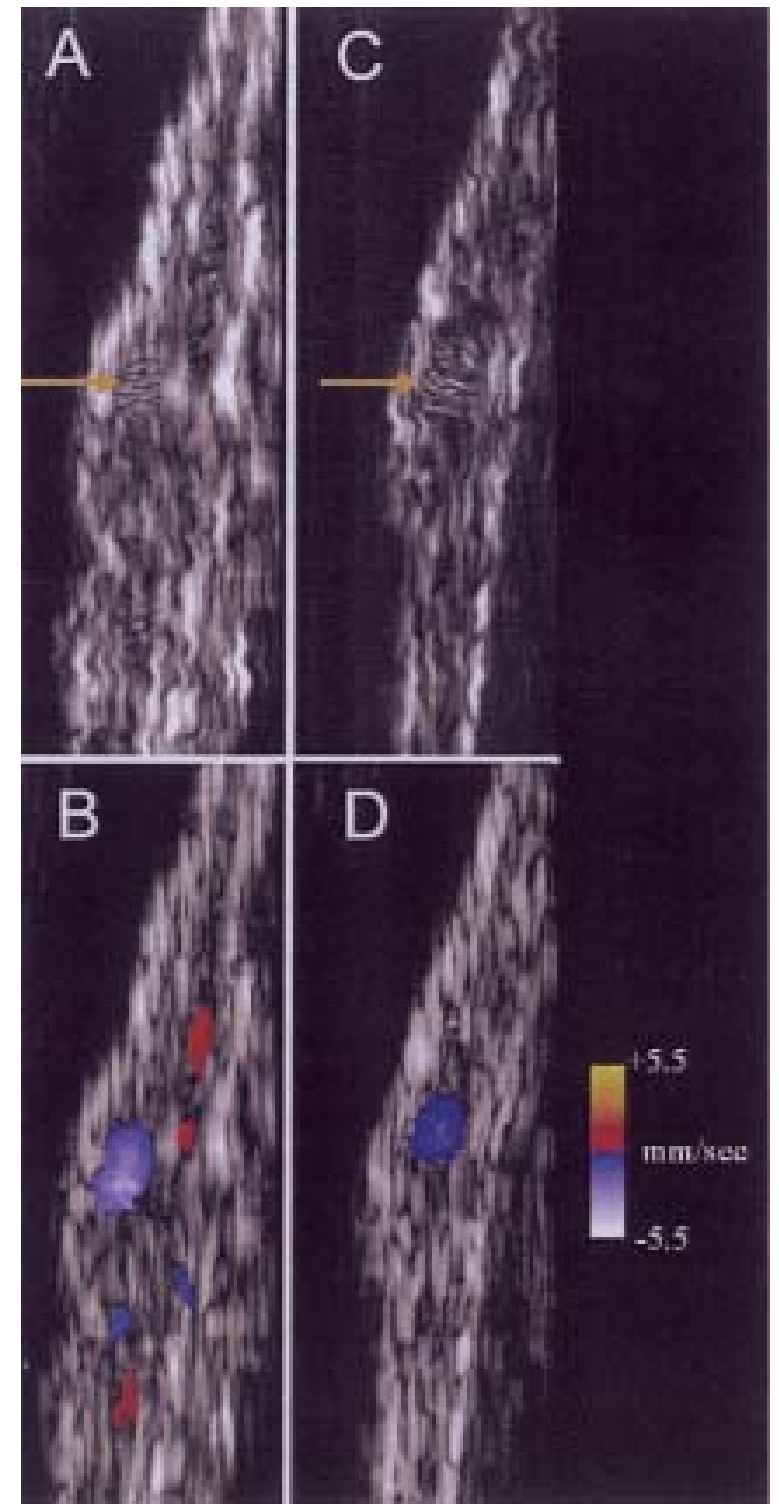

Fig. 8. Swept-scan and color-Doppler images of blood vessels of the rabbit iris, primarily the arterial circle. The artery is indicated by the orange arrows; its diameter is approximately $200 \mu \mathrm{m}$. The transducer is to the left. A: Swept-scan depiction of blood motion parallel to the transducer axis with the eye at $37^{\mathrm{a}} \mathrm{C}$. B: Color-Doppler image equivalent to A. C: Swept-scan depiction at $4^{\mathrm{a} C}$. D: Color-Doppler image equivalent to C. (Printed with the permission of Ronald Silverman, Department of Ophthalmology, Weill Medical College of Cornell University.)

promising multi-feature approach using spectrum analysis and morphometric parameters for discrimination of malignant and benign breast lesions [67]. Feleppa and Machi are extending spectrum-analysis methods to evaluation of sentinel lymph nodes of breast-cancer 
patients for the purpose of guiding micro-histology and immuno-histochemical staining [141,142]. Miller, Holland, and others are evaluating myocardial status using integrated-backscatter and attenuation measures (equivalent to spectral midband parameters) $[8,60,143-$ 145]. Alam, Ophir, Insana, and other investigators are developing elastographic methods for applications in detecting breast and prostate cancers as well as for evaluating them and monitoring the effectiveness of therapy $[37,54,75-78,82,146]$. Additional studies are applying elastographic and scatterer-property techniques to the evaluation of arterial and venous health and microvascular status [55,147-151]. Deng and other investigators are studying the use of contrast agents to detect alterations in normal perfusion that may signal the development of neoplasms through their abnormal neovasculature [105-129]. Lanza, Wickline, and others are using targeted UCAs to deliver therapeutic agents or to detect atheromatous lesions and possibly to deliver therapeutic agents to the lesions [6,7,130-134]. Some preliminary evidence suggests that the neo-vasculature of some cancerous lesions may be targetable [152156]. Ferrara, Silverman, and others are applying advanced methods of flow measurement to detecting and evaluating cancers [135-138]. Obviously, a great deal of research is underway applying these emerging methods to critical issues in disease detection, evaluation, and management across an extremely broad range of important medical needs.

\section{Future technological developments}

Future technological developments in these emerging technologies can be expected to occur in three general areas: advances within each technological area, advances based on combinations of ultrasonic methods with each other, and advances resulting from correlations of information derived by ultrasonic and nonultrasonic methods.

\subsection{Advances within individual emerging ultrasound technologies}

Ultrasound instruments are incorporating a great deal of digital technology in array controls, scanning, gain manipulations, frequency shifting, pulse shaping, speckle suppression, beam formation, dynamic focusing, image enhancement, and the like. Top-of-theline instruments already are incorporating new imaging modes that only recently were advanced research top- ics, e.g. harmonic imaging and VHFU intra-vascular imaging. Advances can be expected in all of these areas. In addition, array technology constantly is improving in terms of fabrication and materials, which results in increased sensitivity, improved focusing, greater bandwidth, and higher quality conventional images. Array technology also is extending into higher frequency ranges and providing superior image definition. All of these enhancements will advance basic ultrasound imaging and the emerging technologies discussed above.

This article summarizes recent developments in spectrum-analysis-based techniques and elastographic techniques. However, to fully utilize the potential of technology based on spectrum analysis and elastographic methods, additional computing power will be required to perform the needed mathematical functions in real time. Improvements will occur by incorporating digital signal-processing capabilities and also by developing newer, more efficient algorithms for processing echo signals. As one example, much of the information extracted from echo signals using Fourier transformation to compute power spectra also may be made available using auto-regression and wavelet methods.

Any method of tissue-type imaging requires a database of parameter values, clinical variables, and tissue types so that, using an appropriate classification method, parameters associated with each tissue type of interest can be established. A lookup table will need to be generated using the optimal classifier for each set of tissues of interest. Instruments intended for use in tissue-type imaging for various organs would then include a battery of lookup tables in which each table provides a means of distinguishing among specific tissue types for a given organ, e.g. cancerous vs. non-cancerous prostate tissue.

\subsection{Advances among emerging ultrasound technologies}

Imaging based on spectrum analysis, elasticity, and ultrasound contrast agents generally provides different information derived from independent properties of tissue. To the extent that information obtained using these methods is truly independent, more powerful classification and tissue-type differentiation may be possible in many applications. 


\subsection{Advances in combinations of ultrasonic and other technologies}

Ultrasonic imaging of any kind is based on the mechanical properties of scatterers in the ultrasound field, i.e., the relative magnitudes and the spatial properties of their acoustical impedances in the case of conventional imaging, spectrum analysis, and advanced flowmeasuring methods. Contrast-agent imaging depends upon the properties of the UCAs themselves. Elastography depicts strains in tissue and, from that, displays relative tissue rigidities over volumes with dimensions that tend to be much larger than a wavelength; however, the estimates of strain are computed using signals backscattered from smaller scatterers within the ultrasound field. Other imaging modalities, such as CT and MR imaging, MR spectroscopy, PET and SPECT imaging, utilize entirely different types of information. Images generated using these methods display spatial distributions of a variety of tissue properties other than the mechanical properties of constituent scatterers. Therefore, if fundamentally independent information from non-ultrasound technologies can be merged with ultrasonically derived information, further substantial improvements in tissue characterization and tissue-type imaging may be possible.

\section{Conclusion}

The emerging ultrasound technologies discussed in this article show exciting potential for detecting early markers of disease, assessing disease status, planning and targeting treatment, and following disease progression and regression in response to treatment.

Spectrum-analysis-based technologies sense the mechanical properties of microscopic scatterers in tissue; elastographic methods depict elasticity properties of tissue on a larger scale; contrast agents enhance bloodcontaining spaces and offer means of assessing flow, perfusion, and the sites of targeted vascular lesions; and advanced flow-measuring methods detect and measure slow flow as well as flow detectable and measurable by conventional methods. Spectrum analysis has demonstrated its potential for distinguishing cancerous from non-cancerous tissues in a variety of organs - most notably the eye, prostate gland, and lymph nodes and also has shown potential for distinguishing among various types of venous thrombi and arterial plaques. While these applications of spectrum analysis require a database for characterizing the tissue types of inter- est, spectrum analysis also can depict changes in tissue that occur over time by sensing changes occurring in constituent scatterers; in such applications, the organ or lesion of interest provides its own baseline reference point for assessing temporal variations.

Elastography clearly presents an enormous potential in cases where a disease process alters the local stiffness or elasticity of tissues. While cancerous lesion detection and evaluation are obvious candidate applications for elastography, detection and characterization of vascular lesions and evaluation of vessel-wall properties may prove to be additional applications for elastography [157].

Contrast agents obviously benefit imaging and assessment of large blood-filled volumes such as the cardiac chambers, arteries, and veins, but their greatest future value may lie in their ability to provide perfusion information, e.g. in assessing cardiac ischemia or in revealing and quantitatively evaluating targetable lesions such as atherosclerotic plaque or neo-vasculature associated with some types of cancers. UCAs take advantage of new techniques of harmonic imaging, but also potentially can enhance the advanced, correlationbased methods of detecting and measuring slow and chaotic flow, which may be of great value in characterizing neo-vasculature associated either with tissue repair or with very early cancerous lesions.

Combining these emerging technologies with each other or fusing the information provided by each of them may provide even more powerful tools for detecting and evaluating disease. In addition, each emerging ultrasound technology potentially can be correlated with other conventional and emerging non-ultrasound technologies to synergize the independent types of information provided by each modality and further extend their medical value.

\section{Acknowledgments}

The work of the authors has been supported in part by NIH grants CA53561, CA84274, CA84588, EB000238, and HL59302.

\section{References}

[1] F.L. Lizzi, M. Greenebaum, E.J. Feleppa, M. Elbaum and D.J. Coleman, Theoretical framework for spectrum analysis in ultrasonic tissue characterization, J Acoust Soc Am 73(4) (1983), 1366-1373. 
[2] E.J. Feleppa, F.L. Lizzi, D.J. Coleman and M.M. Yaremko, Diagnostic spectrum analysis in ophthalmology: A physical perspective, Ultrasound Med Biol 12(8) (1986), 623-631.

[3] F.L. Lizzi, D.L. King, M.C. Rorke, J. Hui, M. Ostromogilsky, M.M. Yaremko, E.J. Feleppa and P. Wai, Comparison of theoretical scattering results and ultrasonic data from clinical liver examinations, Ultrasound Med Biol 14(5) (1988), 377385.

[4] F.L. Lizzi, E.J. Feleppa, M. Astor and A. Kalisz, Statistics of ultrasonic spectral parameters for prostate and liver examinations, IEEE Trans Ultrason Ferro Freq Control 44 (1997), 935-942.

[5] M. O'Donnell, D. Bauwens, J.W. Mimbs and J.G. Miller, Broadband integrated backscatter: an approach to spatially localized tissue characterization in vivo, Proceedings of the IEEE Ultrasonics Symposium 79 CH 1482-1489, 1979, pp. 175-178.

[6] G.M. Lanza, R.L. Trousil, K.D. Wallace, J.H. Rose, C.S. Hall, M.J. Scott, J.G. Miller, P.R. Eisenberg, P.J. Gaffney and S.A. Wickline, In vitro characterization of a novel, tissuetargeted ultrasonic contrast system with acoustic microscopy, $J$ Acoust Soc Am 104 (1998), 3665-3672.

[7] G.M. Lanza, K. Wallace, M.J. Scott, C. Cachetis, D. Abendschein, D. Christy, A. Sharkey, J. Miller, P. Gaffney and S. Wickline, A novel site-targeted ultrasonic contrast agent with broad biomedical applications, Circulation 94 (1996), 3334-3340.

[8] J.G. Miller, J.E. Perez, SJ.A. Wickline, S.L. Baldwin, B. Barzilai, V. Davila-Roman, R.J. Fedewa, A.E. FinchJohnston, C.S. Hall, S.M. Handley, F.D. Huckett, M.R. Holland, A. Kovacs, G.M. Lanza, S.S. Lewis, J.N. Marsh, J. Mobley, D.E. Sosnovik, R.L. Trousil, K.D. Wallace and K.R. Waters, Backscatter imaging and myocardial tissue characterization, Proceedings of the IEEE Ultrasonics Symposium, Sendai Japan, 1998, pp. 1373-1383.

[9] E.J. Feleppa, Physics of diagnostic ultrasound for surgeons, in: Ultrasound for Surgeons, J. Machi and B. Sigel, eds, Igaku-Shoin Medical Publishers, New York, 1996, pp. 7-41.

[10] M.J. Rondeau, R.H. Silverman, I.M. Aslanides, H.O. Lloyd, F.L. Lizzi and D.J. Coleman, Functional ultrasound imaging of the ciliary muscle during accommodation, Invest Ophthalmol Vis Sci 36(4) (1995), S502.

[11] I.M. Aslanides, M.J. Rondeau, R.H. Silverman, D.Z. Reinstein, D.J. Najafi, H.O. Lloyd, R. Ursea, F.L. Lizzi and D.J. Coleman, In-vivo functional ultrasound imaging of ciliary muscle response to pharmacological agents, Invest Ophthalmol Vis Sci 36(4) (1995), S564.

[12] D.J. Coleman, M.J. Rondeau, R.H. Silverman and F.L. Lizzi, Computerized ultrasonic biometry and imaging of intraocular tumors for the monitoring of therapy, Trans Amer Ophth Soc LXXXV (1987), 49-81.

[13] M.L. Oelze and W.D. O'Brien, Method of improved scatterer size estimation and application to parametric imaging using ultrasound, J Acoust Soc Am 112(6) (2002), 3053-3063.

[14] M.L. Oelze, J.F. Zachary and W.D. O'Brien, Characterization of tissue microstructure using ultrasonic backscatter: Theory and technique for optimization using a Gaussian form factor, $J$ Acoust Soc Am 112(3) (2002), 1202-1211.

[15] M.F. Insana, R.F. Wagner, D.G. Brown and T.J. Hall, Describing small-scale structure in random media using pulseecho ultrasound, J Acoust Soc Am 87 (1990), 179-192.

[16] M.F. Insana, J.G. Wood and T.J. Hall, Identifying acoustic scattering sources in normal renal parenchyma in vivo by varying arterial and ureteral pressures, Ultrasound Med Biol 1896(7) (1992), 587-599.

[17] M.F. Insana, J.G. Wood, T.J. Hall, G.G. Cox and L.A. Harrison, Effects of endothelium-1 on renal microvasculature measured using quantitative ultrasound, Ultrasound Med Biol 21 (1995), 1143-1151.

[18] D.J. Coleman, R.H. Silverman, M.J. Rondeau, F.L. Lizzi, I. W. McLean and F.J. Jakobiec, Correlations of acoustic tissue typing of malignant melanoma and histopathologic features as a predictor of death, Amer J Ophth 110 (1990), 380-388.

[19] M.J. Rondeau, R.H. Silverman, D.J. Coleman and F.L. Lizzi, The acoustic microarchitecture of human malignant melanoma, in: Acoustical Imaging, (Vol. 13), L.W. Kessler, ed., Plenum Press, NY, 1987.

[20] D.J. Coleman, F.L. Lizzi, R.H. Silverman, L. Helson, J.H. Torpey and M.J. Rondeau, A model for acoustic characterization of intraocular tumors, Invest Ophth Visual Sci 26 (1985), 545-550.

[21] D.J. Coleman, F.L. Lizzi, R.H. Silverman, R.M. Ellsworth, B.G. Haik, D.H. Abramson, M.E. Smith and M.J. Rondeau, Regression of uveal malignant melanoma following cobalt60 plaque: Correlates between pre-treatment acoustic spectrum analysis and tumor regression, Retina 5 (1985), 73-78.

[22] F.L. Lizzi, D.J. Coleman, L. Franzen, E.J. and Feleppa, Use of a spectrum analysis system for characterization of malignant melanoma, in: Ultrasound in Medicine, D. White and E.A. Lyons, eds, Plenum Press, New York, 1978, pp. 559-561.

[23] E.J. Feleppa, J.A. Ketterling, A. Kalisz, S. Urban, C.R. Porter, J. Gillespie, P.B. Schiff, R.D. Ennis, C.S. Wuu, W. Moul, I.A. Sesterhenn and P.T. Scardino, Prostate imaging based on RF spectrum analysis and non-linear classifiers for guiding biopsies and targeting radiotherapy, in: Medical Imaging 2001: Ultrasonic Imaging and Signal Processing, Society of Photo-Optical Instrumentation Engineers, (Vol. 4325), K.K. Shung and M. Insana, eds, Bellingham, WA, 2001, pp. 371-379.

[24] E.J. Feleppa, R.D. Ennis, P.B. Schiff, C.S. Wuu, A. Kalisz, J. Ketterling, S. Urban, T. Liu, W.R. Fair, C.R. Porter and J.R. Gillespie, Spectrum-analysis and neural networks for imaging to detect and treat prostate cancer, Ultrasonic Imaging 23 (2001), 90-106.

[25] E.J. Feleppa, J.A. Ketterling, A. Kalisz, S. Urban, C.R. Porter, J.W. Gillespie, P.B. Schiff, R.D. Ennis, C.S. Wuu and W.R. Fair, Advanced ultrasonic tissue-typing and imaging based on radio-frequency spectrum analysis and neuralnetwork classification for guidance of therapy and biopsy procedures, in: Proceedings of CARS 2001-Computer Assisted Radiology and Surgery, H. Lemke et al, eds, Amsterdam, 2001, pp. 333-337.

[26] E.J. Feleppa, R.D. Ennis, P.B. Schiff, C.S. Wuu, A. Kalisz, J.A. Ketterling, S. Urban, T. Liu, W.R. Fair, C.R. Porter and J.R. Gillespie, Ultrasonic spectrum-analysis and neuralnetwork classification as a basis for Ultrasonic Imaging to target brachytherapy of prostate cancer, J Brachytherapy Intl 1(1) (2002), 1-6.

[27] E.J. Feleppa, S. Urban, A. Kalisz, C.R. Porter, J. Gillespie, R.D. Ennis, C.S. Wuu and P.B. Schiff, Advances in tissue-type imaging (TTI) for detecting and evaluating prostate cancer, in Proceedings of the 2002 Ultrasonics Symposium, Institute of Electrical and Electronics Engineers, Piscataway, (in press).

[28] C.E. Metz, J.H. Shen and B.A. Herman, New methods for estimating a binormal ROC curve from continuously-distributed test results, Presented at the 1990 Joint Meetings of the Amer- 
ican Statistical Society and the Biometric Society, Anaheim, CA, August 1990.

[29] Y.C. Fung, Biomechanical properties of living tissues, Ch. 7, Springer Verlag, NY, 1981.

[30] T.A. Krouskop, T.M. Wheeler, F. Kallel, B.S. Garra and T. Hall, Elastic moduli of breast and prostate tissues under compression, Ultrason. Imag. 20 (1998), 260-274.

[31] J. Ophir, I. Céspedes, H. Ponnekanti, Y. Yazdi and X. Li, Elastography: A method for imaging the elasticity in biological tissues, Ultrason. Imag. 13 (1991), 111-134.

[32] M. O'Donnell, A.R. Skovoroda, B.M. Shapo and S.Y. Emelianov, Internal displacement and strain imaging using ultrasonic speckle tracking, IEEE Trans. Ultrason. Ferroel. Freq. Contr. UFFC-41 (1994), 314-325.

[33] K.J. Parker, S.R. Huang, R.A. Musulin and R.M. Lerner, Tissue response to mechanical vibrations for sonoelasticity imaging, Ultrasound Med Biol 16 (1990), 241-246.

[34] T.A. Krouskop, D.R. Dougherty and F.S. Vinson, A pulsed Doppler ultrasonic system for making noninvasive measurements of the mechanical properties of soft tissue, J Rehabil. Res Dev 24 (1987), 1-8.

[35] R.M. Lerner, S.R. Huang and K.J. Parker, Sonoelasticity images derived from ultrasound signals in mechanically vibrated tissues, Ultrasound Med Biol 16 (1990), 231-239.

[36] R.M. Lerner and K.J. Parker, Sono-elasticity in ultrasonic tissue characterization and echographic imaging, Proceedings of the 7th Eur. Comm. Workshop, J.M. Thijssen, ed., Nijmegen, The Netherlands, 1987.

[37] B.S. Garra, E.I. Céspedes, J. Ophir, R.S. Spratt, R.A. Zuurbier, C.M. Magnant and M.F. Pennanen, Elastography of breast lesions: Initial clinical results, Radiology 202 (1997), 79-86.

[38] L.S. Taylor, B.C. Porter, D.J. Rubens and K.J. Parker, Three-dimensional sonoelastography: principles and practices, Phys Med Biol 45(6) (2000), 1477-1494.

[39] L. Gao, K.J. Parker, R.M. Lerner and S.F. Levinson, Imaging of the elastic properties of tissue - A review, Ultrasound Med Biol 22 (1996), 959-977.

[40] J. Ophir, S.K. Alam, B. Garra, F. Kallel, E. Konofagou, T. Krouskop and T. Varghese, Elastography: ultrasonic estimation and imaging of the elastic properties of tissues, Proceedings of the Institution of Mechanical Engineers, Part H: Journal of Engineering in Medicine, (Vol. 213), (No. 3), 1999, pp. 203-233.

[41] J. Ophir, I. Céspedes, B. Garra, H. Ponnekanti, Y. Huang and N. Maklad, Elastography: ultrasonic imaging of tissue strain and elastic modulus in vivo, Euro J Ultrasound 3 (1996), 49-70.

[42] P. Chaturvedi, M.F. Insana and T.J. Hall, 2-D companding for noise reduction in strain imaging, IEEE Trans Ultrason Ferroel Freq Control 45 (1998), 179-191.

[43] P. Chaturvedi, M.F. Insana, T.J. Hall and M. Bilgen, 3-D companding using linear arrays for improved strain imaging, Proceedings of the IEEE Ultrasonics Symposium, 1997, pp. 1435-1438.

[44] E.E. Konofagou and J. Ophir, A new elastographic method for estimation and imaging of lateral displacements, lateral strains, corrected axial strains and Poisson's ratios in tissues, Ultrasound Med Biol 24 (1998), 1183-1199.

[45] F. Yeung, S.F. Levinson and K.J. Parker, Non-rigid motion estimation of ultrasound image sequences using an adaptive deformable mesh, (Vol. 3338), Proceedings of the SPIE Medical Imaging 1998, 1998, pp. 806-817.
[46] Y. Zhu, P. Chaturvedi and M.F. Insana, Strain imaging with a deformable mesh, Ultrasonic Imaging 21 (1999), 127-146.

[47] H. Ponnekanti, J. Ophir, Y. Huang and I. Céspedes, Fundamental mechanical limitations on the visualization of elasticity contrast in elastography, Ultrasound Med Biol 21 (1995) 533-543.

[48] F. Kallel, M. Bertrand, J. and Ophir, Fundamental limitations on the contrast-transfer efficiency in elastography: an analytic study, Ultrasound Med Biol 22 (1996), 463-470.

[49] T. Varghese and J. Ophir, A theoretical framework for performance characterization of elastography: the strain filter, IEEE Trans Ultrason Ferroelec Freq Control 44 (1997), 164172.

[50] T. Varghese and J. Ophir, An analysis of elastographic contrast-to-noise ratio, Ultrasound Med Biol 24(6) (1998), 915-24.

[51] R. Righetti, J. Ophir and P. Ktonas, Axial resolution in elastography, Ultrasound Med Biol 28 (2002), 101-113.

[52] S.K. Alam, J. Ophir and T. Varghese, Elastographic resolution criteria: an experimental study, IEEE Trans Ultrason Ferroelectr Freq Control 47(1) (2000), 304-309.

[53] T.J. Hall, M. Bilgen, M.F. Insana and T.A. Krouskop, Phantom materials for elastography, IEEE Trans. Ultrason Ferroel Freq Control 44 (1997), 1355-1365.

[54] F. Kallel, J. Ophir, K. Magee and T. Krouskop, Elastographic imaging of low-contrast elastic modulus distributions in tissue, Ultrasound Med Biol 24 (1998), 409-425.

[55] C.L. DeKorte, E.I. Céspedes, A.F.W. van der Steen and C.T. Lancee, Intravascular elasticity imaging using ultrasound: feasibility studies in phantoms, Ultrasound Med Biol 23 (1997), 735-746.

[56] T. Varghese, M. Bilgen and J. Ophir, Phase aberration effects in elastography, Ultrasound Med Biol 27(6) (2001) 819-827.

[57] R.E. Boucher and J.C. Hassab, Analysis of discrete implementation of generalized cross correlator, Proceedings of the IEEE Trans Acoust Speech Sig 29 (1981), 609-611.

[58] E.I. Céspedes, Y. Huang, J. Ophir and S. Spratt, Methods for estimation of sub-sample time delays of digitized echo signals, Ultrasonic Imaging 17 (1995), 142-171.

[59] S.K. Alam and J. Ophir, The effect of nonlinear signal transformations on bias errors in elastography, IEEE Trans Ultrason Ferroelec Freq Control 47(1) (2000), 297-303.

[60] D.E. Sosnovik, S.L. Baldwin, M.R. Holland and J.G. Miller, Transmural variation of myocardial attenuation and its potential effect on contrast-mediated estimates of regional myocardial perfusion, J Am Soc Echocardiogr 14 (2001), 782788.

[61] F. Forsberg, J.B. Liu, H.J. Chiou, N.M. Rawool, L. Parker and B.B. Goldberg, Comparison of fundamental and wideband harmonic contrast imaging of liver tumors, Ultrasonics $\mathbf{3 8}$ (2000), 110-113.

[62] F. Kallel and J. Ophir, A least squares estimator for elastography, Ultrasonic Imaging 19 (1997), 195-208.

[63] E.I. Céspedes and J. Ophir, Reduction of image noise in elastography, Ultrasonic Imaging 15 (1993), 89-102.

[64] S.K. Alam and J. Ophir, Reduction of signal decorrelation from mechanical compression of tissues by temporal stretching: approaches to elastography, Ultrasound Med Biol 23(1) (1997), 95-105.

[65] S.K. Alam, J. Ophir and E.E. Konofagou, An adaptive strain estimator for elastography, IEEE Trans Ultrason Ferroelec Freq Control 45(2) (1998), 461-472. 
[66] E.E. Konofagou, T. Varghese, J. Ophir and S.K. Alam, Power spectral strain estimators in elastography, Ultrasound Med Biol 25(7) (1999), 1115-1129.

[67] T. Varghese, E.E. Konofagou, J. Ophir, S.K. Alam and M. Bilgen, Direct strain estimation in elastography using spectral cross-correlation, Ultrasound Med Biol (in press).

[68] S.K. Alam, F.L. Lizzi, E.J. Feleppa and T. Varghese, Novel estimators for elastography, in Medical Imaging 2001: Ultrasonic Imaging and Signal Processing, K.K. Shung and M.F. Insana, eds, Proceedings of SPIE, vol. 4325, pp. 314325.

[69] S.K. Alam, F.L. Lizzi, E.J. Feleppa, T. Liu and A. Kalisz, Computer aided diagnosis of breast lesions using a multifeature analysis procedure, in Medical Imaging 2002: Ultrasonic Imaging and Signal Processing, M.F. Insana and W. Walker, eds, Proceedings of SPIE, 2002, vol. 4687, pp. 296303.

[70] T. Varghese, M. Bilgen and J. Ophir, Multi-resolution imaging in elastography, IEEE Trans Ferroel Freq Control $\mathbf{4 5}$ (1998), 65-75.

[71] E.E. Konofagou, J. Ophir, F. Kallel and T. Varghese, Elastographic dynamic range expansion using variable applied strains, Ultrasonic Imaging 19 (1997), 145-166.

[72] J.C. Bamber and N.L. Bush, Freehand elasticity imaging using speckle decorrelation rate, Acoustical Imaging 22 (1996), 285-292.

[73] S. Srinivasan, F. Kallel, R. Souchon and J. Ophir, Analysis of an adaptive strain estimation technique in elastography, Ultrasonic Imaging 24 (2002), 109-118.

[74] A.R. Skovoroda, S.Y. Emelianov and M. O'Donnell, Tissue elasticity reconstruction based on ultrasonic displacement and strain images, IEEE Trans Ultrason Ferroelectr Freq Control 42 (1995), 747-765.

[75] F. Kallel, R.E. Price, E. Konofagou and J. Ophir, Elastographic imaging of the normal canine prostate in vitro, Ultrasonic Imaging 21 (1999), 201-215.

[76] M.M. Doyley, P.M. Meaney and J.C. Bamber, Evaluation of an iterative reconstruction method for quantitative elastography, Phys Med Biol 45(6) (2000), 1521-1540.

[77] P.E. Barbone and J.C. Bamber, Quantitative elasticity imaging: what can and cannot be inferred from strain images, Phys Med Biol 47(12) (2002), 2147-2164.

[78] A. Lorenz, H.J. Sommerfeld, M. Garcia-Schürmann, S. Philippou, T. Senge and H. Ermert, A new system for the acquisition of ultrasonic multicompression strain images of the human prostate in vivo, IEEE Trans Ultrason Ferroelec Freq Control 46 (1998), 1147-1154.

[79] K. Nightingale, M.S. Soo, R. Nightingale and G. Trahey, Acoustic radiation force impulse imaging: in vivo demonstration of clinical feasibility, Ultrasound Med Biol 28(2) (2002), 227-235.

[80] F.L. Lizzi, C.X. Deng, R. Muratore, J.A. Ketterling, S.K. Alam, S. Mikaelian, S.N. Ramachandran, Radiation-force technique to monitor lesions during ultrasonic therapy, $\mathrm{Ul}$ trasound Med Biol (2002) (in press).

[81] F. Viola, D.M. Longo, M.B. Lawrence and W.F. Walker, Analysis of clot formation with acoustic radiation force, in: Medical Imaging 2002: Ultrasonic Imaging and Signal Processing, M.F. Insana and W. Walker, eds, Proceedings of SPIE, vol. 4687, 2002, pp. 235-242.

[82] M. Fatemi and J.F. Greenleaf, Application of radiation force in noncontact measurement of the elastic parameters, Ultrasonic Imaging 21(2) (1999), 147-154.
[83] A. Pesavento, A. Lorenz and H. Ermert, System for real-time elastography, Electron Lett 35 (1999), 941-942.

[84] Y. Zhu and T.J. Hall, A modified block matching method for real-time freehand strain imaging, Ultrason Imaging $\mathbf{2 4}$ (2002), 161-176.

[85] J. Ophir and K.J. Parker, Contrast agents in diagnostic ultrasound, Ultrasound Med Biol 15 (1989), 319-333.

[86] B.B. Goldberg, J.B. Liu and F. Forsberg, Ultrasound contrast agents: a review, Ultrasound Med Biol 20 (1994), 319-333.

[87] B.B. Goldberg, Role of contrast agents in ultrasonography, Appl Radiol (4-7) (October 1997).

[88] K.W. Ferrara, C.R.B. Merritt, P.N. Burns, S. Foster, R.F. Mattrey and S.A. Wickline, Evaluation of tumor angiogenesis with US: imaging, Doppler and contrast agents, Acad Radiol 7 (2000), 824-839.

[89] F.J. Ten Cate and P.R. Silverman, Potential clinical applications of echocardiologic contrast agents for intravenous use, Cardiovasc Imaging 3 (1991), 17-23.

[90] J.D. Kasprzak, B. Paelinck, F.J. Ten Cate, W.B. Vletter, N. de Jong, D. Poldermans, A. Elhendy, A. Bouakaz and J.R. Roelendt, Comparison of native and contrast-enhanced harmonic echocardiography for visualization of left ventricular endocardial border, Am J Cardiol 83 (1999), 211-217.

[91] L. Solbiati, L. Gova, T. Ierace, P. Marelli, V. Osti and S.N. Goldberg, The importance of arterial phase imaging for wideband harmonic sonography for the characterization of focal lesions in liver cirrhosis using a second generation contrast agent, Radiology 217 (2000) (P), 305.

[92] R.P. Kedar, D. Cosgrove, V.R. McGready, J.C. Bamber and E.R. Carter, Microbubble contrast agent for color Doppler US: effect on breast masses, Radiology 198 (1996), 679-686.

[93] S. Huber, T. Helbich, J. Kettenbach, W. Dock, I.V. Zuna and S. Delmore, Effects of a microbubble contrast agent on breast tumors: computer-assisted quantitative assessment with color Doppler US- early experience, Radiology 208 (1998), 485-489.

[94] W.K. Moom, J.G. Im, D.Y. Noh and M.C. Han, Nonpalpable breast lesions: evaluation with Doppler and a microbubble contrast agent-initial experience, Radiology 217 (2000), 240246.

[95] B.B. Goldberg, C.R.B. Merritt, L. Needleman, C.W. Piccoli, F. Forsberg, L. Parker, A. Maitino, D.A. Merton, S.M. Schultz and N.M. Rawool, Contrast enhanced ultrasound and mammography for breast cancer detection, J Ultrasound Med 20 (2001), S95.

[96] H.A. Bogers, J.P.M. Sedelaar, H.P. Beerlage, J.J.M.C.H. DeLaRosette, F.M.J. Debruyne, H. Wijkistra and R.G. Aarnink, Contrast-enhanced three dimensional power Doppler angiography of the human prostate: correlation with biopsy outcome, Urology 54 (1999), 97-104.

[97] F. Frauscher, A. Klauser, E.J. Halpern, W. Horninger and G. Bartsch, Contrast-enhanced color Doppler targeted biopsy: improved detection of prostate cancer, Lancet 357 (2001), 1849-1850.

[98] E.J. Halpern, M. Rosenberg and L.G. Gomella, Prostate cancer: color-enhance US for detection, Radiology 219 (2001), 219-225.

[99] F. Frauscher, A. Klauser, H. Volgger, E.J. Halpern, L. Pallwein, H. Steiner, A. Shuster, W. Horninger, H. Rogatsch and G. Bartsch, Comparison of contrast-enhanced Color Doppler targeted biopsy to conventional systematic biopsy: impact on prostate cancer detection, J Urol 167 (2002), 1648-1652. 
[100] N. de Jong, R. Cornet and C.T. Lancee, Higher harmonics of vibrating gas-filled microspheres. Part two: measurements, Ultrasonics 32 (1994), 455-459.

[101] W.T. Shi, F. Forsberg, A.L. Hall, R.Y. Chiao, J. Liu, S. Miller, K.E. Thomenius, M.A. Wheatley and B.B. Goldberg, Subharmonic imaging with microbubble contrast agents: initial results, Ultrasonic Imaging 21 (1999), 79-94.

[102] W.T. Shi, F. Forsberg, A. Tornes, J. ?stensen and B.B. Goldberg, Destruction of contrast microbubbles and the association with inertial cavitation, Ultrasound Med Biol 26 (2000), 1009-1019

[103] J.E. Chomas, P. Dayton, J. Allen, K. Morgan and K.W. Ferrara, Mechanisms of contrast agent destruction, IEEE Trans Ultrason Ferroel Freq Control 48 (2001), 232-248.

[104] F. Forsberg, Physics of contrast agents, in: Ultrasound Contrast Agents, B.B. Goldberg, ed., Martin Dunitz, London, 1997, pp. 8-20.

[105] C.X. Deng and F.L. Lizzi, A review of physical phenomena associated with ultrasonic contrast agents and illustrative clinical applications, Ultrasound Med Biol 28 (2002), 277-286.

[106] M.J.K. Blomely, T. Albrecht, D.O. Cosgrove, R.J. Eckersley, J. Butler-Barnes, V. Jayaram, N. Patel, R.A. Heckemann, A. Bauer and R. Schlief, Stimulated acoustic emission to image a late liver and spleen-specific phase of Levovist in normal volunteers and patients with and without liver disease, Ultrasound Med Biol 25 (1999), 1341-1352.

[107] C.X. Deng, FL. Lizzi, R.H. Silverman, R. Ursea and D.J. Coleman, Imaging and spectrum analysis of contrast agents in the in vivo rabbit eye using very-high-frequency ultrasound, Ultrasound Med Biol 24 (1998), 383-394.

[108] A. Klibanov, M. Hughes, J. Marsh, C. Hall, J. Miller, J.H. Wible and G. Brandenburger, Targeting of ultrasound contrast agents: An in vitro feasibility study, Acta Radiol 38 (1997), 113-120.

[109] S.M. Demos, H. Alkan-Onyuksel, B.J. Kane, K. Ramani, A. Nagaraj, R. Greene, M. Klegerman and D.D. McPherson, In vivo targeting of acoustically reflective liposomes for intravascular and transvascular ultrasonic enhancement, J Amer College Cardiology 33 (1999), 867-875.

[110] F. Forsberg, J.B. Liu, D.A. Merton, N.M. Rawool and B.B. Goldberg, Parenchymal enhancement and tumor visualization using a new ultrasound contrast agent, J Ultrasound Med 14 (1995), 949-957.

[111] D.P. Frush, D.S. Babcock, K.S. White and L.L. Barr, Quantification of intravenous contrast-enhanced Doppler power spectrum in the rabbit carotid artery, Ultrasound Med Biol 21 (1995), 41-47.

[112] V.F. Duda, G. Rode and R. Schlief, Echocontrast agent enhanced color flow imaging of the breast, Ultrasound Obstet Gynecol 3 (1993), 191-194.

[113] W.T. Shi and F. Forsberg, Ultrasonic characterization of the nonlinear properties of contrast microbubbles, Ultrasound Med Biol 26 (2000), 93-104.

[114] B.A. Schrope and V.L. Newhouse, Second harmonic ultrasonic blood perfusion measurement, Ultrasound Med Biol 19 (1993), 567-579.

[115] P.H. Chang, K.K. Shung, S. Wu and H.B. Levene, Second harmonic imaging and harmonic Doppler measurements with Albunex, IEEE Trans Ultrason Ferroel Freq Control 42 (1995), 1020-1027.

[116] O. Lotsberg, J.M. Hovem and B. Askum, Experimental observation of subharmonic oscillations in infoson bubbles, $J$ Acoust Soc Am 99 (1996), 1366-1369.
[117] P.M. Shankar, P. Dala Krishna and V.L. Newhouse, Advantage of subharmonic over second harmonic backscatter for contrast-to-tissue echo enhancement, Ultrasound Med Biol 24 (1998), 395-399.

[118] P.J.A. Frinking, A. Bouakaz, J. Kirkhorn, F.J. Ten Cate and N. de Jong, Ultrasound contrast imaging: Current and new potential methods, Ultrasound Med Biol 26 (2000), 965-975.

[119] P.J.A. Frinking, E.I. Cespedes, J. Kirkhorn, H.G. Torp and N. de Jong, A new ultrasound contrast imaging approach based on the combination of multiple imaging pulses and a separate release burst, IEEE Trans Ultrason Ferroel Freq Control 48 (2001), 643-651.

[120] D. Hope Simpson, C.T. Chin and P.N. Burn, Pulse inversion Doppler: a new method for detecting nonlinear echoes from microbubble contrast agents, IEEE Trans Ultrason Ferroel Freq Control 46 (1999), 372-382.

[121] T.R. Porter and F. Xie, Transient myocardial contrast after initial exposure to diagnostic ultrasound pressures with minute doses of intravenously injected microbubbles: Demonstration and potential mechanisms, Circulation 92 (1995), 23912395.

[122] X. Verbeek, L.A.F. Ledoux, J.M. Willigers, P.J. Brands and A.P.G. Hoeks, Experimental investigation of the pulse inversion technique for imaging ultrasound contrast agents, $J$ Acoust Soc Am 107 (2000), 2281-2290.

[123] N. Kamiyama, F. Moriyasu, Y. Mine and Y. Goto, Analysis of flash echo from contrast agent fro designing optimal ultrasound diagnostic systems, Ultrasound Med Biol 25(3) (1999), 411-420.

[124] T. Albrecht, M.J. Bromley, D.O. Cosgrove, S.D. TaylorRobinson, V. Jayaram, R. Echersley, A. Urbank, J. ButlerBarnes and N. Patel, Non-invasive diagnosis of hepatic cirrhosis by transit-time analysis of an ultrasound contrast agent, Lancet 353 (1999), 1579-1583.

[125] K.Q. Schwarz, G.P. Bezante, X. Chen and R. Schlief, Quantitative echo contrast concentration measurement by Doppler sonography, Ultrasound Med Biol 19 (1993), 289-297.

[126] K. Wei, A.R. Jayaweera, S. Firoozan, A. Linka, D.M. Skyba and S. Kaul, Quantification of myocardial blood flow with ultrasound-induced destruction of microbubbles administered as a constant venous infusion, Circulation 95(5) (1998), 473-483.

[127] C.X. Deng, F.L. Lizzi, A. Rosado, R.H. Silverman and D.J. Coleman, Measurement of volumetric flow rate with ultrasonic contrast agents using a dual-frequency-band technique, The 25th International Symposium on Ultrasonic Imaging and Tissue Characterization 2000, Arlington, VA, May 2000.

[128] C.M. Sehgal and P.H. Arger, Mathematical modeling of the dilution curves for ultrasonographic contrast agents, J Ultrasound Med 7 (1997), 471-479.

[129] C.X. Deng, J.A. Ketterling, F.L. Lizzi, R.H. Silverman and D.J. Coleman, Measurement of flow rates with ultrasonic contrast agents using a depletion method. The 26th International Symposium on Ultrasonic Imaging and Tissue Characterization 2001, Arlington, VA, May 2001.

[130] J.R. Linder, J. Song, J. Christiansen, A.L. Klibanov, F. Xu and K. Ley, Ultrasound assessment of inflammation and renal tissue injury with microbubbles targeted to P-selectin, Circulation 104 (2001), 2107-2112.

[131] E.D. Unger, T.P. McCreey and R. Sweitzer, Ultrasound enhances gene expression of liposomal transfection, Radiology 32 (1997), 723-727.

[132] J.N. Marsh, C.S. Hall, M.J. Scott, R.W. Fuhrhop, P.J. Gaffney, S.A. Wickline and G.M. Lanza, Improvements in the ultra- 
sonic contrast of targeted perfluorocarbon nanoparticles using an acoustic transmission line model, IEEE Trans Ultrason Ferroelectr Freq Control 49(1) (2002), 29-38.

[133] G.M. Lanza and S.A. Wicklinem, Targeted ultrasonic contrast agents for molecular imaging and therapy, Prog Cardiovasc Dis 44(1) (2001), 13-31.

[134] C.S. Hall, J.N. Marsh, M.J. Scott, P.J. Gaffney, S.A. Wickline and G.M. Lanza, Temperature dependence of ultrasonic enhancement with a site-targeted contrast agent, J Acoust Soc Am 110(3 Pt 1) (2001), 1677-1684.

[135] R.H. Silverman, R. Folberg, H.C. Boldt, H.O. Lloyd, M.J. Rondeau, M.G. Mehaffey, F.L. Lizzi and D.J. Coleman, Correlation of ultrasound parameter imaging with microcirculatory patterns in uveal melanoma, Ultrasound Med Biol 23 (1997), 573-581.

[136] R.H. Silverman, R. Ursea, D. Kruse, K.W. Ferrara, M.J. Rondeau and D.J. Coleman, Ultrasound measurement of thermoregulation of microperfusion in the eye, Ultrasound Med Biol 28 (2002), 1500-1515.

[137] R.H. Silverman, F.L. Lizzi, B.G. Ursea, M.J. Rondeau, N.B. Eldeen, A. Kalisz, H.O. Lloyd and D.J. Coleman, Highresolution Ultrasonic Imaging and characterization of the ciliary body, Invest Ophthalmol Vis Sci 42 (2001), 885-894.

[138] R.H. Silverman, D. Kruse, D.J. Coleman and K.W. Ferrara, High-resolution Ultrasonic Imaging of blood-flow in the anterior segment of the eye, Invest Ophthalmol Vis Sci $\mathbf{4 0}$ (1999), 1373-1381.

[139] D. Kruse, J. Fornaris, R. Silverman, D. Coleman and K. Ferrara, A swept-scanning mode for estimation of blood velocity in the microvasculature, IEEE Trans Ultrason Ferro Freq Control 45 (1998), 1437-1440.

[140] R. Ursea, D.J. Coleman, R.H. Silverman, F.L. Lizzi and W. Harrison, Correlation of high-frequency ultrasound backscatter with tumor microstructure in iris melanoma, Ophthalmology 105 (1998), 906-912.

[141] T. Noritomi, J. Machi, E.J. Feleppa, E. Yanagihara and K. Shirouzu, In vitro investigation of lymph node metastasis of colorectal cancer using ultrasonic spectral parameters, Ultrasound Med Biol 24 (1998), 235-243.

[142] T. Tateishi, J. Machi, E.J. Feleppa, R.H. Oishi, J. Jucha, E. Yanagihara, L.J. McCarthy, T. Noritomi and K. Shirouzu, In vitro diagnosis of axillary lymph node metastases in breast cancer by spectrum analysis of radio frequency echo signals, Ultrasound Med Biol 24(8) (1998), 1151-1159.

[143] D.E. Sosnovik, S.L. Baldwin, S.H. Lewis, M.R. Holland and J.G. Miller, Transmural variation of myocardial attenuation measured with a clinical imager, Ultrasound Med Biol 27 (2001), 1643-1650.

[144] P.K. Tamirisa, M.R. Holland, J.G. Miller and J.E. Perez, U1trasonic tissue characterization of hypertrophic left ventricu- lar myocardium, Echocardiography 18 (2001), 593-597.

[145] J.E. Perez and J.G. Miller, Myocardial tissue characterization: An extension of echocardiography, in: Imaging in Cardiovascular Disease, G.M. Pohost, ed., Lippincott Williams \& Wilkins, Philadelphia, 2000, pp. 115-120.

[146] D.J. Rubens, M.A. Hadley, S.K. Alam, L. Gao, R.D. Mayer and K.J. Parker, Sonoelasticity imaging of prostate cancer: in vitro results, Radiology 195 (1995), 379-383.

[147] C.L. de Korte, A.F. van der Steen, E.I. Cespedes and G. Pasterkamp, Intravascular ultrasound elastography in human arteries: initial experience in vitro, Ultrasound Med Biol 24(3) (1998), 401-408.

[148] C.L. de Korte and A.F. van der Steen, Intravascular ultrasound elastography: an overview, Ultrasonics 40(1-8) (2002), 859965.

[149] S.Y. Emelianov, X. Chen, M. O’Donnell, B. Knipp, D. Myers, T.W. Wakefield and J.M. Rubin, Triplex ultrasound: elasticity imaging to age deep venous thrombosis, Ultrasound Med Biol 28(6) (2002), 757-767.

[150] M.F. Insana and T.J. Hall, Parametric ultrasound imaging from backscatter coefficient measurements: image formation and interpretation, Ultrasonic Imaging 12 (1990), 245-267.

[151] M.F. Insana, T.J. Hall, J.G. Wood and Y. Zhong-Yu, Renal ultrasound using parametric imaging techniques to detect changes in microstructure and function, Invest Radiol $\mathbf{2 8}(8)$ (1993), 720-723.

[152] A. Uchida, D.S. O'Keefe, D.J. Bacich, P.L. Molloy and W.D. Heston, In vivo suicide gene therapy model using a newly discovered prostate-specific membrane antigen promoter/enhancer: a potential alternative approach to androgen deprivation therapy, Urology 58(2 Suppl 1) (2001), 132-139.

[153] F. Watt, A. Martorana, D.E. Brookes, T. Ho, E. Kingsley, D.S. O'Keefe, P.J. Russell, W.D. Heston and P.L. Molloy, A tissue-specific enhancer of the prostate-specific membrane antigen gene, FOLH1, Genomics 1;73(3) (2001), 243-254.

[154] S.S. Chang, V.E. Reuter, W.D. Heston and P.B. Gaudin, Metastatic renal cell carcinoma neovasculature expresses prostate-specific membrane antigen, Urology 57(4) (2001), 801-805.

[155] D.J. Bacich, J.T. Pinto, W.P. Tong and W.D. Heston, Cloning, expression, genomic localization and enzymatic activities of the mouse homolog of prostate-specific membrane antigen/NAALADase/folate hydrolase, Mamm Genome 12(2) (2001), 117-123.

[156] D.S. O'Keefe, A. Uchida, D.J. Bacich, F.B. Watt, A. Martorana, P.L. Molloy and W.D. Heston, Prostate-specific suicide gene therapy using the prostate-specific membrane antigen promoter and enhancer, Prostate 45(2) (2000), 149-157.

[157] C.L. de Korte and A.F. van der Steen, Intravascular ultrasound elastography: an overview, Ultrasonics 40 (2002), 859-865. 


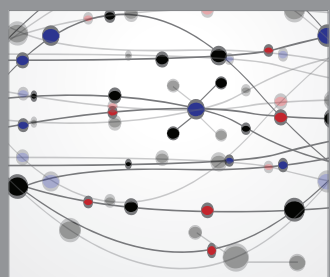

The Scientific World Journal
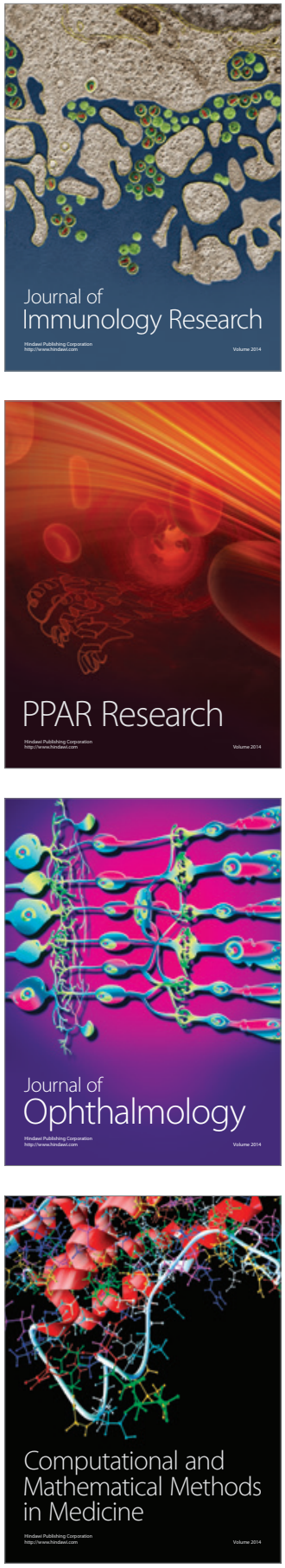

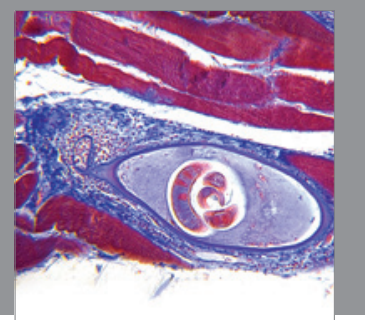

Gastroenterology

Research and Practice
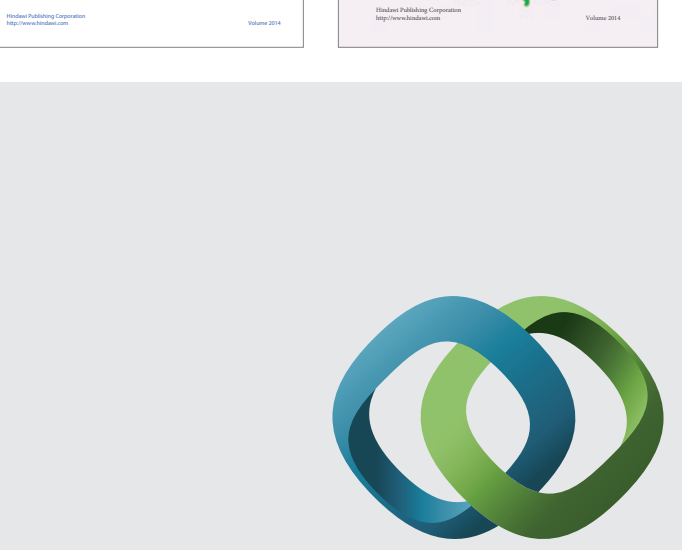

\section{Hindawi}

Submit your manuscripts at

http://www.hindawi.com
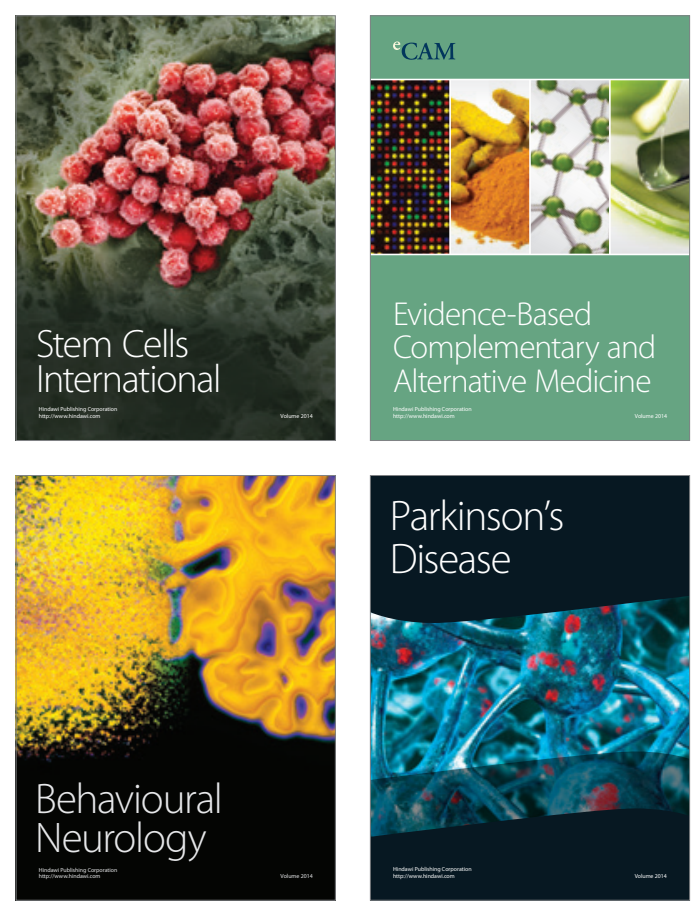

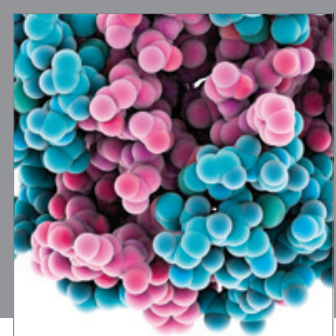

Journal of
Diabetes Research

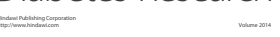

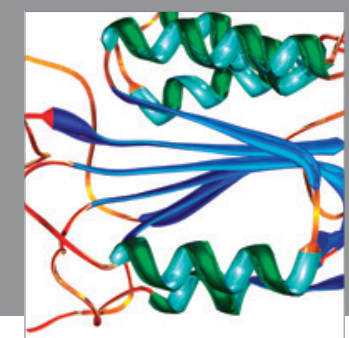

Disease Markers
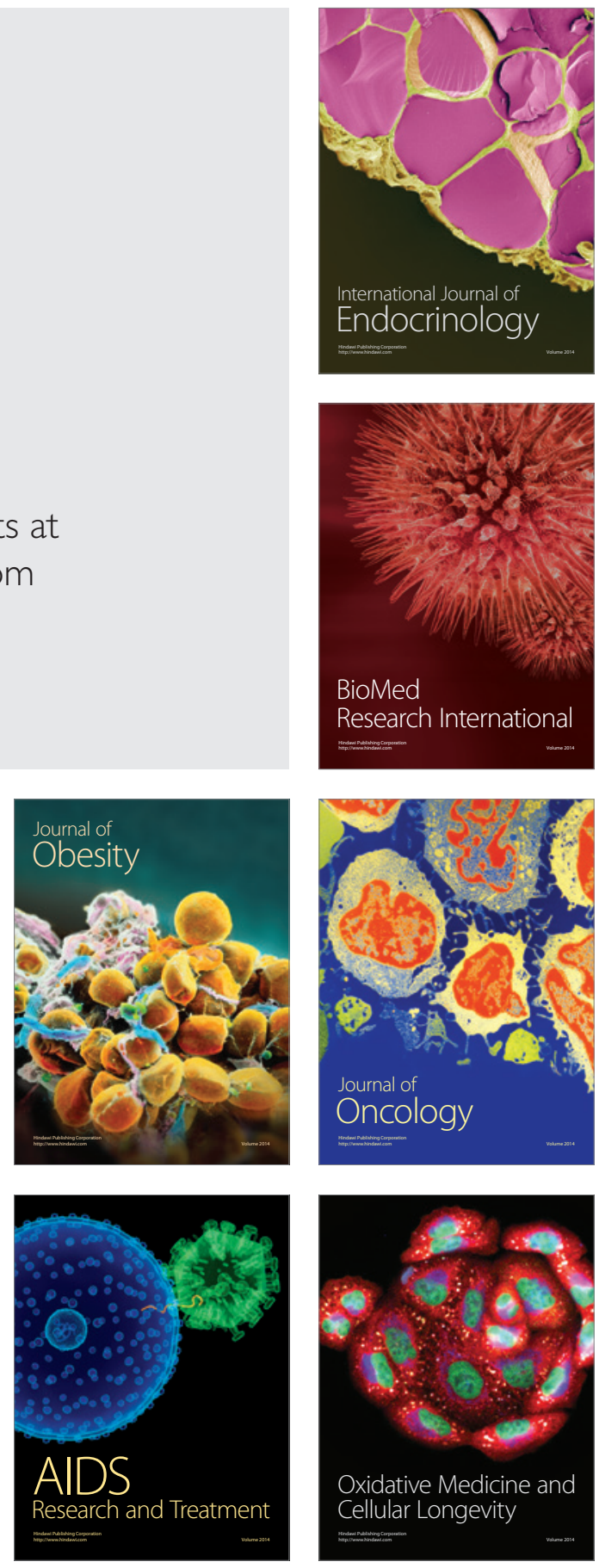